\title{
Hidden in Plain Sight: A Massive, Dusty Starburst in a Galaxy Protocluster at $z=5.7$ in the COSMOS Field
}

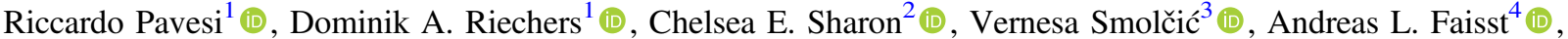 \\ Eva Schinnerer ${ }^{5}$ (i), Christopher L. Carilli ${ }^{6,7}$ (i), Peter L. Capak ${ }^{4}$, Nick Scoville ${ }^{8}$ (i), and Gordon J. Stacey ${ }^{1}$ \\ ${ }_{1}^{1}$ Department of Astronomy, Cornell University, Space Sciences Building, Ithaca, NY 14853, USA; rp462@ cornell.edu \\ ${ }^{2}$ Department of Physics \& Astronomy, McMaster University, 1280 Main Street West, Hamilton, ON L85-4M1, Canada \\ ${ }^{3}$ University of Zagreb, Physics Department, Bijenička cesta 32, 10002 Zagreb, Croatia \\ ${ }^{4}$ Infrared Processing and Analysis Center, California Institute of Technology, Pasadena, CA 91125, USA \\ ${ }^{5}$ Max Planck Institute for Astronomy, Königstuhl 17, D-69117, Heidelberg, Germany \\ ${ }^{6}$ National Radio Astronomy Observatory, P.O. Box O, Socorro, NM 87801, USA \\ ${ }^{7}$ Cavendish Astrophysics Group, University of Cambridge, Cambridge, CB3 OHE, UK \\ ${ }^{8}$ Astronomy Department, California Institute of Technology, MC 249-17, 1200 East California Boulevard, Pasadena, CA 91125, USA \\ Received 2018 March 21; revised 2018 May 15; accepted 2018 May 19; published 2018 June 29
}

\begin{abstract}
We report the serendipitous discovery of a dusty, starbursting galaxy at $z=5.667$ (hereafter called CRLE) in close physical association with the "normal" main-sequence galaxy HZ10 at $z=5.654$. CRLE was identified by detection of [C II], [N II], and $\mathrm{CO}(2-1)$ line emission, making it the highest-redshift, most luminous starburst in the COSMOS field. This massive, dusty galaxy appears to be forming stars at a rate of at least $1500 M_{\odot} \mathrm{yr}^{-1}$ in a compact region only $\sim 3 \mathrm{kpc}$ in diameter. The dynamical and dust emission properties of CRLE suggest an ongoing merger driving the starburst, which is in a potentially intermediate stage relative to other known dusty galaxies at the same epoch. The ratio of [C II] to [N II] may suggest that an important $(\sim 15 \%)$ contribution to the [C II] emission comes from a diffuse ionized gas component, which could be more extended than the dense, starbursting gas. CRLE appears to be located in a significant galaxy overdensity at the same redshift, potentially associated with a large-scale cosmic structure recently identified in a Lyman $\alpha$-emitter survey. This overdensity suggests that CRLE and HZ10 reside in a protocluster environment, offering the tantalizing opportunity to study the effect of a massive starburst on protocluster star formation. Our findings support the interpretation that a significant fraction of the earliest galaxy formation may occur from the inside out, within the central regions of the most massive halos, while rapidly evolving into the massive galaxy clusters observed in the local universe.
\end{abstract}

Key words: cosmology: observations - galaxies: formation - galaxies: high-redshift - galaxies: ISM - galaxies: starburst - radio lines: galaxies

\section{Introduction}

While a significant fraction of the visible sky is covered by moderate-redshift, low-mass galaxies, the view of dustobscured star formation in the submillimeter sky preferentially selects high-mass, dusty star-forming galaxies (DSFGs) at high redshift. This complementary view of the universe can provide unique insights into galaxy formation processes (e.g., Blain et al. 2002; Casey et al. 2014). While the general population of DSFGs was found to predominantly occupy the peak epoch of cosmic star formation at $z=2-3$, a significant tail of higherredshift, and often brighter, examples appears to already be in place at $z>5$ (e.g., Riechers et al. 2010, 2013, 2017; Walter et al. 2012; Weiß et al. 2013; Strandet et al. 2016, 2017). These submillimeter-selected galaxies in the early universe are often extreme "hyper-starbursts," reaching infrared luminosities of $L_{\mathrm{IR}}>10^{13} L_{\odot}$ and star formation rates (SFRs) exceeding $1000 M_{\odot} \mathrm{yr}^{-1}$ within small spatial regions of a few kiloparsecs in diameter. They are likely the result of major mergers and/or extreme gas accretion events, which may only be possible in the highest-density regions of the early universe (e.g., Capak et al. 2011; Ivison et al. 2011, 2013; Riechers et al. 2011, 2017; Oteo et al. 2016; Marrone et al. 2018).

Recent single-dish studies of the evolution of the submillimeter luminosity function have suggested that, while the space density of DSFGs may significantly decrease at $z>3$, the knee of the luminosity function appears to shift to higher infrared luminosities, perhaps capturing the high-redshift tail of "titans" (e.g., Koprowski et al. 2017). While the $z>4$ infrared luminosity function is far from accurately constrained, and the diversity of processes that may produce dusty hyperstarbursts are not completely understood (e.g., Narayanan et al. 2015), these results may not be completely surprising in the context of recent theoretical work exploring the role of protocluster star formation (e.g., Chiang et al. 2013, 2017). In particular, simulations suggest that a large fraction of earlyuniverse star formation may have taken place in the densest regions, traced by the most massive halos, in an inside-out fashion. That is, a significant fraction of galaxy formation may have started in protocluster cores where the gas densities and galaxy merger probability would have been highest, implying that this star formation may have been bursty and highly dustobscured, as seen in high-redshift DSFGs. The importance of the connection between DSFGs and galaxy overdensities has been known for some time (e.g., Aravena et al. 2010). Even the first $z>5$ DSFG known, AzTEC-3 at $z=5.3$, was quickly recognized to be located near the center of a rich galaxy protocluster (Capak et al. 2011; Riechers et al. 2014a). Recent studies have explored the connection between DSFGs and galaxy overdensities in detail, finding evidence for a strong relationship, potentially getting stronger with redshift toward the early universe (e.g., Lewis et al. 2017; Smolčić et al. 2017a). Two notable case studies of clustered, dusty galaxy formation are the extremely rich protocluster of DSFGs 
identified at $z=4$ by Oteo et al. (2018) and the surprisingly high incidence $(4 / 25)$ of very close association $(<600 \mathrm{kpc})$ of DSFGs to $z>6$ quasars (Decarli et al. 2017). The former case shows that several protocluster members may be experiencing a dusty starburst phase simultaneously, perhaps triggered by a massive gas flow. Both cases show that active galactic nuclei (AGN) or massive starburst galaxies may be found in association and can be serendipitously discovered in sensitive Atacama Large (sub-)Millimeter Array (ALMA) observations.

Here we describe the serendipitous discovery of the brightest and highest-redshift dusty starburst in COSMOS (Scoville et al. 2007) at J2000 $10^{\mathrm{h}} 0^{\mathrm{m}} 59^{\mathrm{s}} .2,1^{\circ} 33^{\prime} 6{ }^{\prime \prime} 6$, which we identified in ALMA observations of atomic fine-structure lines in a closeby galaxy, HZ10. This new DSFG is located only $13^{\prime \prime}(\sim 77 \mathrm{kpc})$ away from HZ10, which is an above average dusty but "normal" (i.e., $\sim L_{\mathrm{UV}}^{*}$ ) galaxy at $z=5.654$ (Capak et al. 2015; Pavesi et al. 2016). HZ10 is a Lyman $\alpha$ emitter (LAE; Murayama et al. 2007) and Lyman break galaxy (LBG) that was selected for [C II] $158 \mu \mathrm{m}$ and dust emission observations with ALMA based on its strong ultraviolet absorption features (Capak et al. 2015). We subsequently followed up HZ10 in [N II] $205 \mu \mathrm{m}$ emission and CO(2-1) (R. Pavesi et al. 2018, in preparation). These observations provide additional support to the interpretation that HZ10 appears to show a more "mature" and metal-rich interstellar medium (ISM) than other "typical" massive galaxies at $z>5$ (Pavesi et al. 2016; Faisst et al. 2017). The new DSFG is also detected in Herschel observations. Its "red" color in the SPIRE 250-500 $\mu \mathrm{m}$ bands is consistent with its high redshift. We refer to this galaxy as COSMOS (FIR-)red line emitter (CRLE, read "curly") in the following, after the methods that lead to its discovery.

In Section 2, we describe the spectroscopic observations that allowed the identification of CRLE as a dusty starburst at $z \sim 5.7$. In Section 3, we present the results of our line measurements and discuss their implications. In Section 4, we present continuum measurements and discuss the dust properties of CRLE and the evidence for a galaxy merger. In Section 5, we analyze public galaxy catalogs to identify and characterize a galaxy overdensity around CRLE, providing evidence for a galaxy protocluster. We conclude in Section 6. An obstacle to any previous identification of this source may have been the presence of an unrelated foreground $(z \sim 0.3)$, low-mass spiral galaxy, which covers the optical counterpart of $\mathrm{CRLE}^{9}$ and therefore prevents detection in the visible and near-IR (NIR) bands. In Appendix A, we detail our constraints on the potential contribution of gravitational lensing from the foreground galaxy to the observed properties of CRLE, finding that lensing may be negligible. Appendix B details our attempt to separate the emission from CRLE from that of the foreground galaxy at observed-frame NIR wavelengths. Appendix $\mathrm{C}$ contains a description of the UVspace dynamical modeling technique we utilized to investigate the $[\mathrm{C} \mathrm{II}]$ observations from CRLE. In this work, we adopt a Chabrier IMF and a flat, $\Lambda$ CDM cosmology with $H_{0}=70 \mathrm{~km} \mathrm{~s}^{-1} \mathrm{Mpc}^{-1}$ and $\Omega_{\mathrm{M}}=0.3$. Quoted lengths are proper sizes unless otherwise specified (for comoving distances).

\footnotetext{
9 For this reason, and for its high submillimeter flux, CRLE may be considered "hidden in plain sight."
}

\section{Observations}

\subsection{ALMA Observations of [C II] and [N II]}

The ALMA data containing the [C II] line for CRLE were first presented by Capak et al. (2015) as Cycle 1 observations of HZ10. A subsequent reprocessing of those data, together with a description of part of the Cycle 3 [N II] observations, was described in detail by Pavesi et al. (2016).

The Cycle 1 observations of [C II] were taken on 2013 November 16 in band 7 as part of a larger project (ID: 2012.1.00523.S; Capak et al. 2015). The pointing resulted in 56 minutes on-source time with 25 usable antennas. The primary beam attenuation factor at the position of CRLE is $\sim 3$. The correlator was set up to target the expected frequency of the [C II] line in $\mathrm{HZ10}$ at $284.835 \mathrm{GHz}$ and provide continuous coverage of the continuum emission in adjacent spectral windows (centered at $\sim 2,12$, and $14 \mathrm{GHz}$ above) with channels of $15.6 \mathrm{MHz}$ width in time division mode (TDM). The synthesized beam size is approximately 0 " $6 \times 0$ ". 5 when adopting natural baseline weighting. We refer to Pavesi et al. (2016) for a complete description of the observations and imaging product.

Cycle 3 observations of [N II] $205 \mu \mathrm{m}$ targeting HZ10 and covering CRLE were taken on 2016 January 1 and 5 in band 6 as part of two separate programs (2015.1.00928.S and 2015.1.00388.S; PIs: Pavesi and Lu, respectively) with one track each in a compact configuration (max. baseline $\sim 300 \mathrm{~m}$ ). The two sets of observations resulted in 50 and 35 minutes on source with $\sim 41-45$ usable $12 \mathrm{~m}$ antennas under good weather conditions at $1.3 \mathrm{~mm}$. The first set of observations was previously described by Pavesi et al. (2016). For the second set of observations, the nearby radio quasar J0948+0022 was observed regularly for amplitude and phase gain calibration, $\mathrm{J} 1058+0133$ was observed for bandpass calibration, and Callisto was used for flux calibration. We estimate the overall accuracy of the flux calibration to be within $\sim 10 \%$. The correlator was set up to cover two spectral windows of $1.875 \mathrm{GHz}$ bandwidth each at $15.6 \mathrm{MHz}\left(\sim 20 \mathrm{~km} \mathrm{~s}^{-1}\right)$ resolution (dual polarization) in TDM in each sideband.

We used the Common Astronomy Software Application (CASA) version 4.5 for data reduction and analysis. We combined data from all observations and produced all images with the CLEAN algorithm, using natural weighting for maximal sensitivity. Imaging these [N II] data results in a synthesized beam size of 1 !! $6 \times 1$ !" 2 at the redshifted [N II] frequency of CRLE and in the continuum map. The rms noise in the phase center is $\sim 0.14 \mathrm{mJy}_{\text {beam }}^{-1}$ in a $44 \mathrm{~km} \mathrm{~s}^{-1}$ wide channel. The final rms noise when averaging over the line-free spectral windows (i.e., over a total $7.1 \mathrm{GHz}$ of bandwidth) is $\sim 18 \mu \mathrm{Jy}$ beam $^{-1}$. The primary beam attenuation factor at the position of CRLE is $\sim 1.8$.

\subsection{VLA Observations of $\mathrm{CO}(2-1)$}

We observed the $\mathrm{CO}(2-1)$ transition line in CRLE $\left(\nu_{\text {rest }}=\right.$ $230.538 \mathrm{GHz}$, redshifted to $\sim 34.58 \mathrm{GHz}$ at $z \sim 5.667$ ), using NSF's Karl G. Jansky Very Large Array (VLA) in the Ka band (project ID: 17A-011, PI: Pavesi). Observations were carried out between 2017 March 4 and April 6, with 27 antennas in the most compact array configuration (D; max. baseline $\sim 950 \mathrm{~m}$ ) under good to moderate weather conditions at $35 \mathrm{GHz}$ (precipitable water vapor columns of 3-6 mm) for eight 

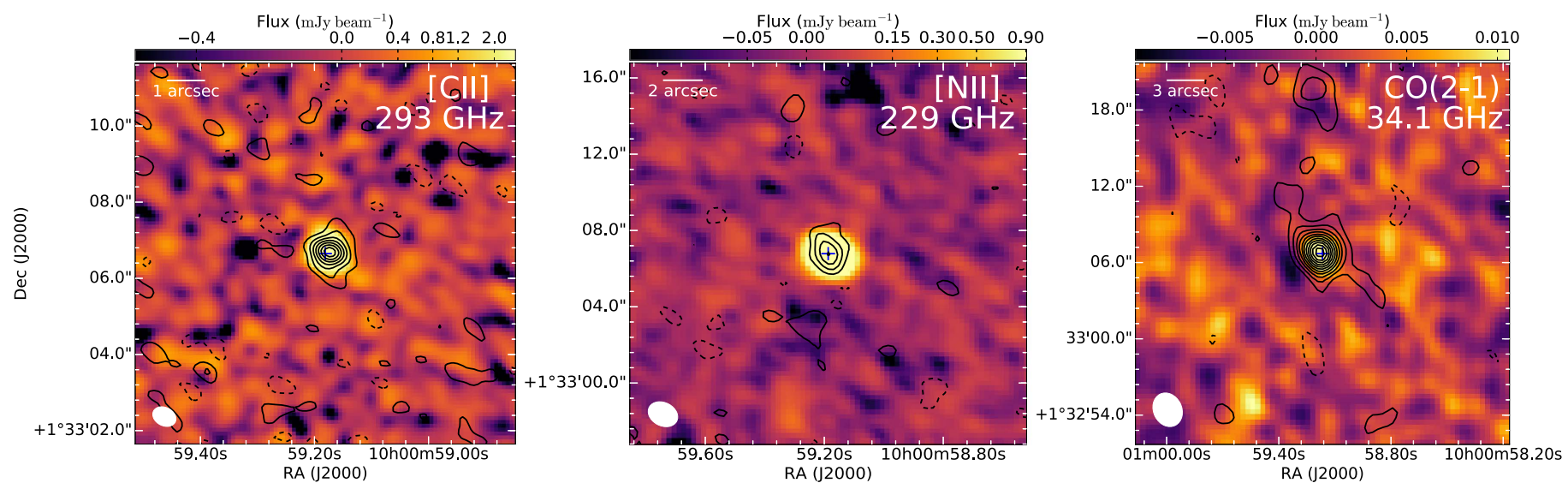

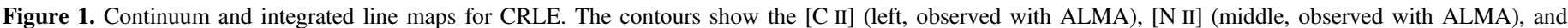

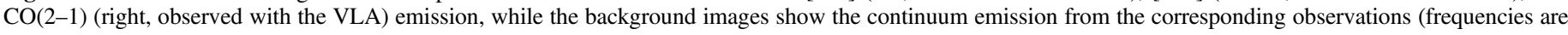

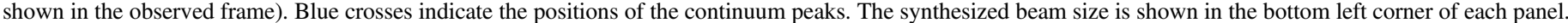

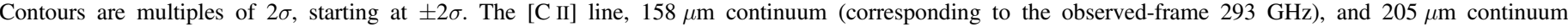
(corresponding to the observed-frame $229 \mathrm{GHz}$ ) emission are resolved. The [N II] line emission is marginally resolved.

sessions. The pointing for these observations was centered on HZ10. The primary beam attenuation factor at the position of CRLE is $\sim 1.08$. The combination of all data results in a total on-source time of $19.8 \mathrm{hr}$.

The nearby radio quasar $\mathrm{J} 1041+0610$ was observed regularly for amplitude and phase gain calibration. Also, 3C286 was observed for bandpass and flux calibration. We estimate the overall accuracy of the flux calibration to be within $\sim 10 \%$, since the phase calibrator flux was measured to be constant within $<5 \%$ across all sessions.

The VLA correlator was utilized in 8 bit mode for maximum sensitivity. In the first three sessions, the correlator was set up with two intermediate-frequency bands (IFs) of eight 128 $\mathrm{MHz}$-wide spectral windows each, centering one IF on the redshifted $\mathrm{CO}(2-1)$ line frequency in $\mathrm{HZ10}$ and the other contiguously below to provide additional continuum sensitivity. The lower IF was moved in the remaining five sessions by centering it on the $\mathrm{CO}(2-1)$ line in CRLE (which otherwise fell onto a subband gap). While these overlapping sidebands limit the simultaneous bandwidth and hence the continuum sensitivity, they provide uninterrupted coverage of the $\mathrm{CO}(2-1)$ line in both galaxies. The channels in all spectral windows were chosen to provide $1 \mathrm{MHz}\left(\sim 9 \mathrm{~km} \mathrm{~s}^{-1}\right)$ resolution (dual polarization) in each IF, to obtain a simultaneous bandwidth of $2.048 \mathrm{GHz}$ for the first three sessions and $1.349 \mathrm{GHz}$ for the remaining five sessions. Because of overlapping spectral coverage, the measurements in the two IFs are not independent. Therefore, we never combine their data but rather only use the line IF for the analysis of CRLE.

We used CASA version 4.7 for data reduction and analysis. We calibrated the visibilities using the scripted VLA pipeline, supplemented by manual flagging through inspection of standard visibility plots. We combined data from all observations and imaged them with the CLEAN algorithm, using natural weighting for maximal sensitivity. The imaging of the $\mathrm{CO}(2-1)$ data results in a synthesized beam size of 2 ! $7 \times 2$ !! 3 at the redshifted $\mathrm{CO}(2-1)$ frequency and in the continuum map. The rms noise in the phase center is $\sim 45 \mu \mathrm{Jy}$ beam $^{-1}$ in a $35 \mathrm{~km} \mathrm{~s}^{-1}$ wide channel. The final rms noise when averaging over the line-free $1.92 \mathrm{GHz}$ of bandwidth is $\sim 2.7 \mu \mathrm{Jy}$ beam $^{-1}$.

\section{Line Emission Properties}

\subsection{Results}

We detect [C II], [N II] $205 \mu \mathrm{m}$, and $\mathrm{CO}(2-1)$ line emission and the adjacent continuum toward CRLE at high significance ( $>8 \sigma$; Figure 1 ), which provides an unambiguous redshift identification. Although a foreground galaxy $\left(z_{\text {phot }} \sim 0.3\right)$ is located along the line of sight to CRLE, potentially causing gravitational lensing of its emission, in Appendix A we constrain the likely magnitude of this effect to be minor $(<10 \%$ flux magnification and negligible spatial distortion). Therefore, we simply extract aperture spectra and fit double Gaussians to the spectral profiles to measure the intrinsic line properties (Figure 2). We report the line properties in Table 1, while the continuum fluxes are listed together with the archival photometric measurements in Table 2.

We measure the deconvolved [C II] spatial FWHM size from UV-plane modeling (see Appendix C) to be 0 ." $46 \pm 0$." 08 , which corresponds to $2.7 \pm 0.5 \mathrm{kpc}$ at $z=5.667$. Using an isotropic virial estimator (Engel et al. 2010) and assuming a [C II] single-Gaussian fit line FWHM of $640 \pm 60 \mathrm{~km} \mathrm{~s}^{-1}$ (which is also compatible with the broad velocity component), we derive a dynamical mass of $(1.5 \pm 0.4) \times 10^{11} M_{\odot}{ }^{10}$

The [N II] emission is only slightly resolved. Using the CASA task UVMODELFIT, we measure a deconvolved FWHM major axis size of 0 .' $98 \pm 0$." 18 , corresponding to $5.8 \pm 1.1 \mathrm{kpc}$. We use the same technique to fit the size of the [C II] emission to provide an accurate comparison to the [N II] emission size, obtaining a result that is compatible with our more sophisticated UV-plane modeling. The [N II] line emission appears to be marginally more extended (formally by a factor of $2.1 \pm 0.5$ ) than the [C II] emission, but higher resolution, and higher signalto-noise [N II] observations are necessary to confirm this finding. In particular, a manual inspection of the UV-radial profile of the [N II] line visibilities appears compatible with the size of the [C II] emission. Neither the $\mathrm{CO}$ line nor the adjacent continuum emission are resolved. We fit the size of the continuum emission at 158 and $205 \mu \mathrm{m}$ in the UV plane, and they are compatible with

\footnotetext{
${ }^{10}$ We note that this estimate is subject to systematic uncertainties of the order of a factor of a few. In particular, a disklike gas distribution would require an inclination correction.
} 


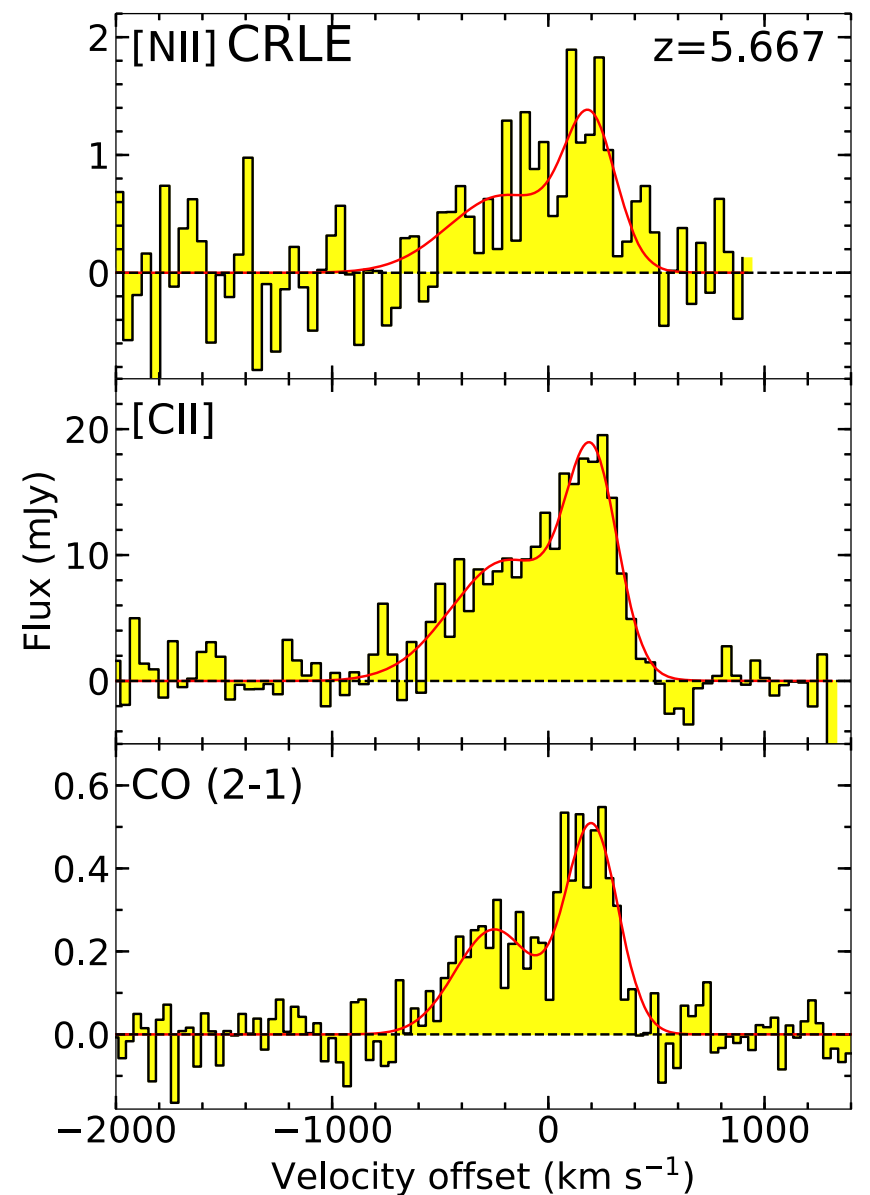

Figure 2. The $[\mathrm{N} \mathrm{II}],[\mathrm{C} \mathrm{II}]$, and $\mathrm{CO}(2-1)$ continuum-subtracted, apertureintegrated line spectra for CRLE (histograms). Double Gaussian fits to the line emission are shown as red curves. A mean redshift of $z=5.667$ was adopted as the velocity reference. The signal-to-noise ratio of the [N II] line does not allow fitting the central frequencies and the widths for the two velocity components. Therefore, these were fixed to the values measured for [C II]. The channel velocity widths are $\sim 43, \sim 44$, and $\sim 35 \mathrm{~km} \mathrm{~s}^{-1}$ for the [N II], [C II], and $\mathrm{CO}(2-1)$ lines, respectively.

the same deconvolved size of $(0$ ". $39 \pm 0$." 01$) \times(0$ ". $31 \pm 0$ ". 02$)$. This implies that the size of the [C II]-emitting region is more extended than the size of the dust continuum by $\sim(30 \% \pm 20 \%)$ in linear dimensions, suggesting that the observed dust continuum may not represent the full extent of the star-forming gas distribution (see also, e.g., Riechers et al. 2014a; Chen et al. 2017).

We find a $[\mathrm{C} \mathrm{II}] /[\mathrm{N}$ II $]$ line luminosity ratio of $19 \pm 4$ in CRLE, which is comparable to the line ratio in the $z=5.3$ DSFG AzTEC-3 of $22 \pm 8$ (Pavesi et al. 2016). This line ratio is sensitive to the fraction of [C II] emission coming from ionized gas, rather than photon-dominated regions (PDRs), because the ionization energy of nitrogen, in contrast to that of carbon, is higher than that of hydrogen. This makes it a tracer of ionized gas only, and the $[\mathrm{C} \mathrm{II}] /[\mathrm{N} \mathrm{III}]$ ratio is approximately constant in ionized gas (e.g., Oberst et al. 2006; Pavesi et al. 2016). Assuming a line ratio of $3 \pm 0.5$ in the ionized gas (Díaz-Santos et al. 2017), we calculate a fraction of the [C II] emission from PDRs of $84 \% \pm 4 \%$ for CRLE and $86 \% \pm 5 \%$ for AzTEC-3.

\subsection{Origin of the $[\mathrm{CII}]$ Emission}

CRLE exhibits approximately the same [C II]/[N II] ratio as AzTEC-3 (Pavesi et al. 2016). This ratio is compatible with the range observed in local (ultra)luminous infrared galaxies ((U)LIRGs). However, given the higher gas density and SFR surface density in high-redshift DSFGs relative to most local (U)LIRGs, one might expect a lower fraction of the [C II] emission to come from the diffuse ionized gas, which is traced by [N II], relative to atomic and molecular PDRs and therefore a higher [C II]/[N II] ratio (Pavesi et al. 2016). Therefore, our observed line ratio suggests that high-redshift DSFGs may host a significant reservoir of diffuse ionized gas, which may be more extended than the starbursting gas. The potentially larger size of the [N II] emitting region in CRLE relative to [C II] (although with significant uncertainty) would provide support for this interpretation if confirmed. Recent measurements of both [N II] fine-structure lines at $205 \mu \mathrm{m}$ (studied here) and $122 \mu \mathrm{m}$ in local galaxies also support the interpretation of the [N II] emission predominantly coming from diffuse ionized gas, rather than compact H II regions (e.g., Herrera-Camus et al. 2016, 2018a, 2018b; Díaz-Santos et al. 2017).

A well-known decreasing trend of the $[\mathrm{C} \mathrm{II}] / L_{\mathrm{IR}}$ ratio with infrared luminosity surface density (a proxy for FUV field strength) offers insight into the origin of the [C II] emission. In particular, a decreasing trend is expected for PDR emission due to the saturation of [C II] line emission at higher FUV field strengths (e.g., Stacey et al. 1991). This trend is clearly observed in a sample of local DSFGs from the Great Observatories All-sky LIRG Survey (GOALS; DíazSantos et al. 2017). However, at the calculated infrared luminosity surface density for CRLE and AzTEC-3, their observed $[\mathrm{C} \mathrm{II}] / L_{\mathrm{IR}}$ ratios $\left(\sim 3-4 \times 10^{-4}\right)$ lie significantly above the best fit to the relation for the GOALS sample (Figure 3). This may suggest an additional contribution besides PDRs to the [C II] emission. If we assume that the PDR contribution to the [C II] luminosity is uniquely determined by the intensity of the radiation field as traced by the $L_{\mathrm{IR}}$ surface density, a more significant contribution from diffuse ionized gas may be a reason for this excess. Furthermore, the fraction of [C II] coming from PDR, rather than ionized gas (as traced by the $[\mathrm{C} \mathrm{II}] /[\mathrm{N} \mathrm{II}]$ ratio), appears to be lower for CRLE and AzTEC-3 for their modeled FIR color, relative to the fitted relation for the GOALS sample (Figure 3). This quantifies the finding that the [C II]/[N II] ratio appears to be low for the more extreme (i.e., higher dust temperature; Figure 3) gas conditions observed in these $z>5$ DSFGs relative to local starbursts (Pavesi et al. 2016). As such, it appears to point toward a larger contribution from diffuse ionized gas to the [C II] emission than what is expected from local starbursts. This diffuse ionized gas may take the form of a gas halo, more extended than the starforming region. We suggest that a potentially larger and more diffuse ionized gas component at $z>5$ may be due to freshly accreted inflowing gas, which is expected to play an important role in powering starbursts in the first billion years of cosmic time. Metallicity is unlikely to play a significant role in affecting the $[\mathrm{C} \mathrm{II}] /[\mathrm{N} \mathrm{II}]$ line ratio, as we constrain $\alpha_{\mathrm{CO}}$ to be low, and the high dust mass for CRLE (see below) likely implies a solar or super-solar metallicity (Bolatto et al. 2013). Nonetheless, an important caveat to our conclusions is that variations in the carbon-to-nitrogen abundance ratio may affect our interpretation of the [N II] line emission properties. 
Table 1

Measured Line Properties of CRLE

\begin{tabular}{|c|c|c|c|}
\hline & [C II] & {$[\mathrm{N} \mathrm{II]}$} & $\mathrm{CO}(2-1)$ \\
\hline \multicolumn{4}{|l|}{ Component 1} \\
\hline$\nu_{\mathrm{obs}}(\mathrm{GHz})$ & $284.892 \pm 0.013$ & $219.0298^{\mathrm{a}}$ & $34.5604 \pm 0.0017$ \\
\hline$S_{\text {peak }}(\mathrm{mJy})$ & $16 \pm 3$ & $1.2 \pm 0.2$ & $0.49 \pm 0.04$ \\
\hline$I\left(\mathrm{Jy} \mathrm{km} \mathrm{s}^{-1}\right)$ & $4.5 \pm 0.9$ & $0.33 \pm 0.07$ & $0.14 \pm 0.02$ \\
\hline \multicolumn{4}{|l|}{ Component 2} \\
\hline FWHM $\left(\mathrm{km} \mathrm{s}^{-1}\right)$ & $610 \pm 140$ & $610^{\mathrm{a}}$ & $420 \pm 90$ \\
\hline$I\left(\mathrm{Jy} \mathrm{km} \mathrm{s}^{-1}\right)$ & $6.3 \pm 1.5$ & $0.40 \pm 0.13$ & $0.11 \pm 0.03$ \\
\hline \multicolumn{4}{|l|}{ Total } \\
\hline Mean redshift & $5.6666 \pm 0.0008$ & & \\
\hline$I\left(\mathrm{Jy} \mathrm{km} \mathrm{s}^{-1}\right)$ & $10.8 \pm 1.2$ & $0.73 \pm 0.15$ & $0.26 \pm 0.02$ \\
\hline$L\left(10^{7} L_{\odot}\right)$ & $930 \pm 100$ & $49 \pm 10$ & $2.7 \pm 0.2$ \\
\hline
\end{tabular}

Notes. We produced integrated line maps over the line FWHM and used CASA to fit a 2D Gaussian model. Then, we extracted aperture spectra utilizing the FWHM of the spatial Gaussian model, correcting the total flux by a factor of 2 to account for the flux outside the aperture. The deconvolved emission size was measured from Gaussian fitting to the visibilities.

${ }^{\text {a }}$ Fixed to the parameter values derived from the [C II] line.

Table 2

Measured Continuum Fluxes (Foreground Galaxy and CRLE)

\begin{tabular}{|c|c|c|}
\hline Wavelength $(\mu \mathrm{m})$ & Flux $(\mu \mathrm{Jy})$ & Band \\
\hline 0.4816 & $4.73 \pm 0.03$ & $H S C g$ \\
\hline 0.6234 & $10.49 \pm 0.03$ & $H S C r$ \\
\hline 0.7740 & $13.38 \pm 0.04$ & $H S C i$ \\
\hline 0.9125 & $17.44 \pm 0.06$ & $H S C z$ \\
\hline 0.9780 & $17.15 \pm 0.12$ & $H S C Y$ \\
\hline 1.0552 & $15.15 \pm 0.07$ & $H S T / \mathrm{F} 105 \mathrm{~W}$ \\
\hline 1.2501 & $17.20 \pm 0.06$ & HST/F125W \\
\hline 1.5418 & $20.67 \pm 0.08$ & $H S T / \mathrm{F} 160 \mathrm{~W}$ \\
\hline 3.6 & $16.29 \pm 0.17$ & Spitzer/IRAC \\
\hline 4.5 & $15.86 \pm 0.18$ & $\cdots$ \\
\hline 5.8 & $19 \pm 5$ & $\ldots$ \\
\hline 8.0 & $21 \pm 4$ & $\cdots$ \\
\hline 24 & $70 \pm 15$ & Spitzer/MIPS \\
\hline $100^{\mathrm{a}}$ & $<5000$ & Herschel/PACS \\
\hline $160^{\mathrm{a}}$ & $<10,000$ & $\ldots$ \\
\hline $250^{\mathrm{a}}$ & $12,000 \pm 900$ & Herschel/SPIRE \\
\hline $350^{\mathrm{a}}$ & $20,900 \pm 1,300$ & $\ldots$ \\
\hline $500^{\mathrm{a}}$ & $31,100 \pm 1,400$ & $\cdots$ \\
\hline $850^{\mathrm{a}}$ & $16,700 \pm 2,000$ & SCUBA-2 \\
\hline $1024(292.8 \mathrm{GHz})^{\mathrm{a}}$ & $16,500 \pm 900$ & ALMA \\
\hline $1308(229.2 \mathrm{GHz})^{\mathrm{a}}$ & $8650 \pm 300$ & $\cdots$ \\
\hline $8800(34.069 \mathrm{GHz})^{\mathrm{a}}$ & $22 \pm 2$ & VLA \\
\hline
\end{tabular}

Notes. The HST/WFC3 fluxes are measured with SExtractor using the Kron mode. We report the Herschel/PACS nondetection as $3 \sigma$ upper limits (Lutz et al. 2011). The SCUBA-2 flux was measured from the S2CLS images (Geach et al. 2017), and the other fluxes were obtained from the COSMOS2015 catalog (Laigle et al. 2016).

${ }^{a}$ Measurements expected to be dominated by emission from CRLE. Additional fluxes may be found in the publicly available COSMOS2015 catalog.

\section{Spectral Energy Distribution Analysis}

\subsection{Optical-to-NIR SED}

CRLE is located behind a local foreground spiral galaxy with a photometric redshift of $\sim 0.35$ and stellar mass of $3.3 \times 10^{9} M_{\odot}$ in the COSMOS2015 catalog (Laigle et al. 2016), which outshines its optical emission. We analyze the HST/WFC3 data targeting HZ10 (Barišić et al. 2017) and measure the NIR fluxes for the foreground galaxy in the $Y, J$, and $H$ bands, potentially including a contribution from CRLE (Figure 4). We measure the fluxes in the HST images, adopting Kron fluxes as measured by SExtractor (Table 2; Bertin \& Arnouts 1996). We then fit the foreground galaxy with Galfit (Peng et al. 2002) and find a faint residual flux to the west of its center. We fix the model to be an exponential light profile with an axis ratio of 0.5 and fit for center position, size, and flux. The residual flux in the $H$-band data corresponds to only $\sim 1 \%-$ $4 \%$ of the total emission, which may be associated with CRLE or an imperfect fit to the central regions of the foreground galaxy (see Appendix B for further details). The foreground galaxy is therefore expected to dominate the total emission at optical-to-NIR wavelengths, at least up to observed-frame $\sim 2 \mu \mathrm{m}$. In order to constrain the contribution of CRLE to the total emission in the near-to-mid-IR, we use Cigale to carry out spectral energy distribution (SED) fitting of the optical and NIR emission (Noll et al. 2009; Serra et al. 2011). We fit the optical and NIR stellar emission with a delayed star formation history (with the ages of the oldest stars ranging from $250 \mathrm{Myr}$ to $12 \mathrm{Gyr}$ and an $e$-folding time ranging from $50 \mathrm{Myr}$ to $8 \mathrm{Gyr}$ ), stellar population synthesis models with metallicity ranging from $1 / 50 Z_{\odot}$ to $Z_{\odot}$ according to Bruzual \& Charlot (2003), and a dust attenuation law according to Calzetti (2001). We determine a stellar mass of $(3.0 \pm 0.5) \times 10^{9} M_{\odot}$ and an SFR of $\sim 1.5 M_{\odot} \mathrm{yr}^{-1}$ for the foreground galaxy, confirming the 

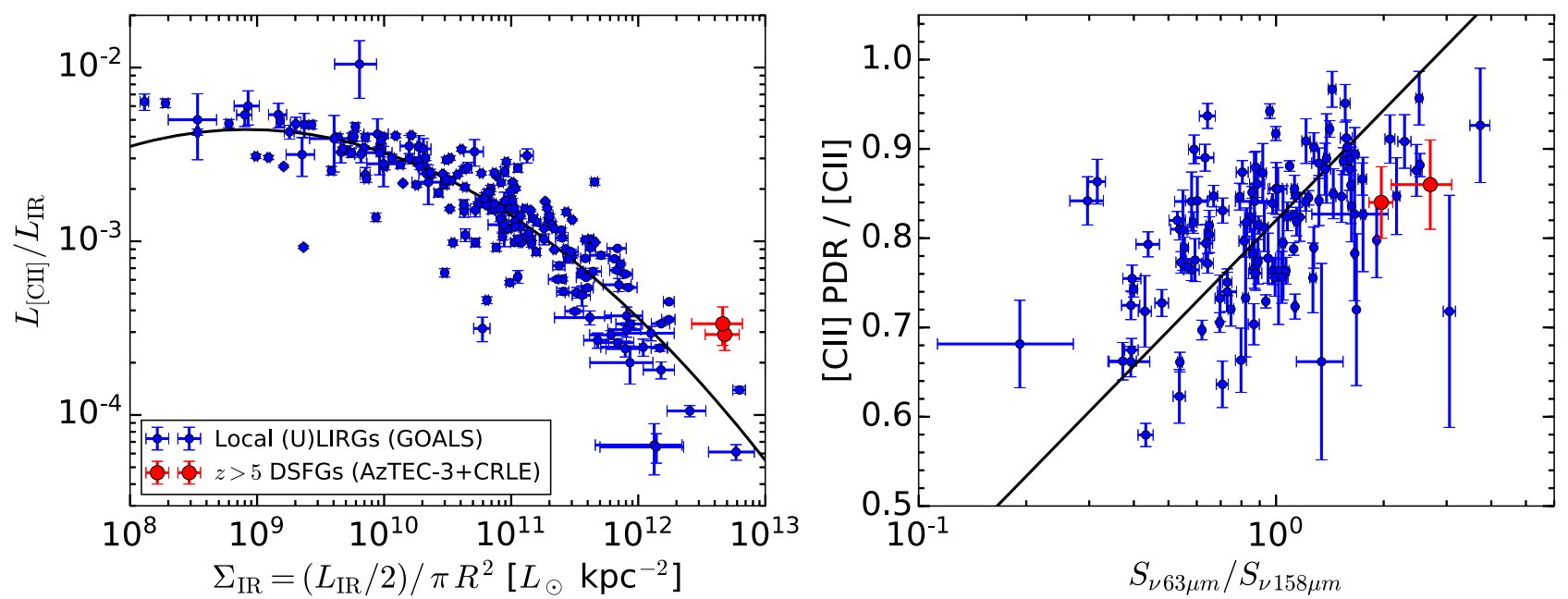

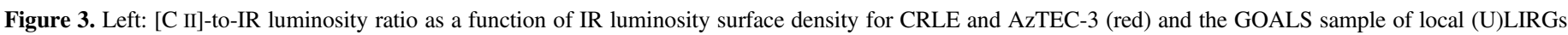

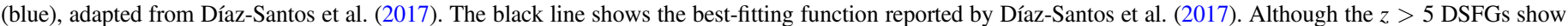

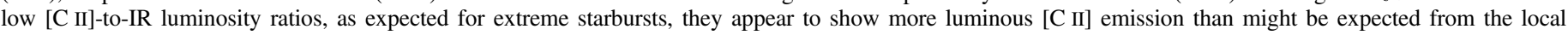

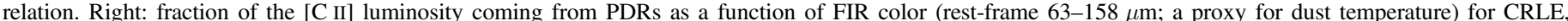

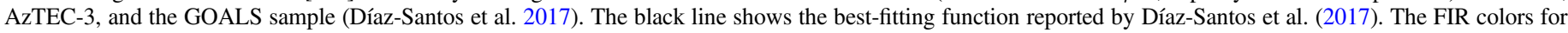

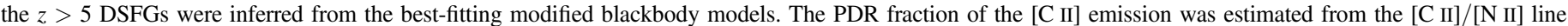

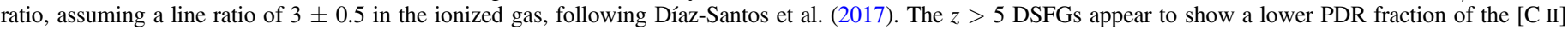
emission relative to the local trend with dust temperature.

catalog stellar mass value. We use the best-fitting Cigale models to constrain the FIR luminosity of the foreground galaxy by adopting dust emission models according to Draine \& Li (2007). We find approximate predictions for the Herschel/SPIRE fluxes of 3.7, 2.0, and $0.8 \mathrm{mJy}$ in the 250 , 350 , and $500 \mu \mathrm{m}$ bands, respectively. These are significantly lower than the measured fluxes in these bands. Therefore, we consider the FIR emission from the foreground galaxy to be subdominant to the emission from CRLE (Table 2).

The Spitzer/IRAC 5.8 and $8.0 \mu \mathrm{m}$ and MIPS $24 \mu \mathrm{m}$ fluxes are not fit well by the available SED models (Figure 4). We interpret this to be an indication of significant contamination by emission from CRLE toward the longer wavelengths. Although the IRAC1 and IRAC2 fluxes are compatible with expectations, the higher IRAC3 and IRAC4 fluxes are difficult to reproduce with the adopted dust emission models (Draine \& Li 2007). The best-fitting SED models were obtained by either including or excluding the MIPS $24 \mu \mathrm{m}$ flux in the fitting (Figure 4). We find that the best-fitting models to the IRAC points overpredict the $24 \mu \mathrm{m}$ flux when excluded, and that the best-fitting models that include the $24 \mu \mathrm{m}$ measurement require strong PAH emission to fit the IRAC4 flux and underpredict the IRAC3 flux at $5.8 \mu \mathrm{m}$ by at least a factor of $\sim 2$. Even artificially reducing the measurement uncertainty on the IRAC3 flux does not improve the fit to this measurement. While it is unclear if the $24 \mu \mathrm{m}$ flux is dominated by emission from CRLE or from the foreground galaxy, we interpret these results as suggesting that perhaps a significant fraction of the flux at $5.8 \mu \mathrm{m}$ may be due to CRLE. A potential caveat to this conclusion may arise in case the foreground galaxy contains an AGN, which could contribute additional flux at these wavelengths, and is presently unconstrained. We use Cigale to derive approximate constraints to the stellar mass in CRLE. If all of the IRAC3 and IRAC4 emission were due to CRLE, the implied stellar mass would be of order $\sim 1-2 \times 10^{11} M_{\odot}$. This likely provides an upper limit to the actual stellar mass of CRLE. A contribution of $\sim 25 \%-50 \%$ of the emission may be plausible, yielding a best stellar mass estimate in the range of $\sim 2.5-10 \times 10^{10} M_{\odot}$.

\subsection{FIR SED and Modified Blackbody Fitting}

In order to model the FIR SED of CRLE as measured by Herschel, ALMA, and JCMT/SCUBA-2, we use a modified blackbody smoothly connected to a mid-IR power law. We use emcee (Foreman-Mackey et al. 2013) to explore the posterior probability distribution of the parameters shown in Figure 5. We employ uniform, nonconstraining priors for the parameters (i.e., flux normalization at rest frame $158 \mu \mathrm{m}$, dust temperature $T_{d}$, dust emissivity parameter $\beta$, rest-frame wavelength at which the optical depth becomes unity $\lambda_{0}$, and mid-IR power-law index $\alpha$; Table 3). By integrating between 42.5 and $122.5 \mu \mathrm{m}$, we find a FIR luminosity of $L_{\mathrm{FIR}}=(1.55 \pm$ $0.05) \times 10^{13} L_{\odot}$. By integrating between 8 and $1000 \mu \mathrm{m}$, we derive an IR luminosity of $L_{\mathrm{IR}}=(3.2 \pm 0.3) \times 10^{13} L_{\odot}$. Because the SED of CRLE is not constrained at mid-IR wavelengths, we follow the standard practice of estimating the SFR based on the FIR luminosity only, to provide a comparison to other $z>5$ DSFGs (Riechers et al. 2014a). By adopting the standard conversion based on a Chabrier IMF (Carilli \& Walter 2013), we infer an SFR of (1550 \pm 50) $M_{\odot} \mathrm{yr}^{-1}$, with the caveat that the real SFR may be up to a factor of $\sim 2 \times$ higher. Given the high level of dust obscuration in CRLE, we cannot exclude the presence of an obscured AGN. However, we expect that an AGN would only introduce minor contributions to the rest-frame $>42.5 \mu \mathrm{m}$ luminosity given the high dust-obscured SFR of CRLE (see, e.g., Kirkpatrick et al. 2015). As an example, the dust-obscured AGN in the $z=4.05$ DSFG GN20 may dominate the mid-IR luminosity, but its contribution to the total IR luminosity appears to be minor (Riechers et al. 2014b). Even for luminous high-redshift quasars, the contribution from AGN-heated hot dust to the FIR luminosity does not appear to exceed $\sim 20 \%$ (Leipski et al. 2013, 2014). By adopting standard (although 

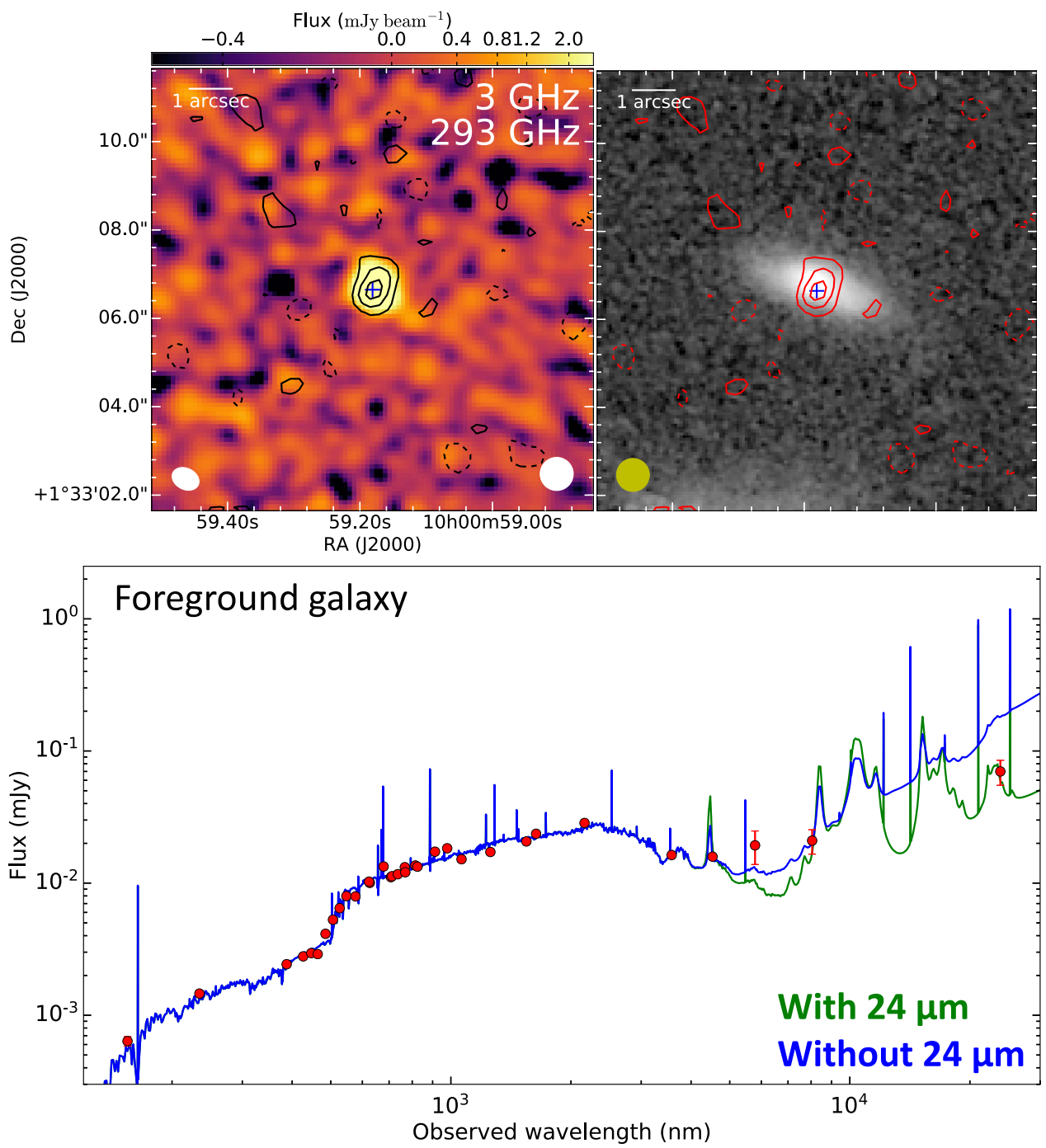

Figure 4. Top: $3 \mathrm{GHz}$ observed-frame continuum emission from CRLE (contours; Smolčić et al. 2017b) on top of ALMA dust continuum from CRLE (left) and NIR emission from the foreground galaxy (right). Contours are shown in $2 \sigma$ steps, starting from $\pm 2 \sigma$. The blue cross represents the $158 \mu \mathrm{m}$ dust emission peak. Top left: color scale showing the rest-frame $158 \mu \mathrm{m}$ continuum (corresponding to observed-frame $293 \mathrm{GHz}$ ). The beam sizes shown are for the $293 \mathrm{GHz}$ data (left) and $3 \mathrm{GHz}$ data (right). Top right: gray-scale HST/WFC3 F160W image from Barišić et al. (2017), showing the foreground disk galaxy at a photometric redshift $z \sim 0.35$. The contribution from CRLE cannot reliably be separated from the foreground galaxy at these wavelengths. Bottom: UV-NIR SED of the foreground galaxy and CRLE, fitted with Cigale models. The models do not provide a good fit to the measurement at $5.7 \mu \mathrm{m}$, suggesting that emission from CRLE may contribute a nonnegligible fraction of, at least, the $5.7 \mu \mathrm{m}$ flux.

uncertain) assumptions from Dunne et al. (2003), we find an estimated dust mass of $(1.3 \pm 0.3) \times 10^{9} M_{\odot}$.

The dust SED shape for CRLE appears to be intermediate between that of AzTEC-3 $(z \sim 5.3)$ and ADFS-27 $(z \sim 5.7$; Figure 5; Riechers et al. 2014a, 2017). We also compare the measured FIR SED of CRLE to model templates of selected lowand high-redshift starbursts spanning a range of physical conditions. A comparison to the template for local starbursts Arp 220 and M82 shows that CRLE resembles M82 more closely, suggesting a comparatively low dust optical depth ${ }^{11}$ (Silva et al. 1998). A comparison to the ultraluminous $z=6.34$ DSFG HFLS3 shows that CRLE appears to have a significantly lower dust temperature (Riechers et al. 2013; Ivison et al. 2016), as evidenced by the longer wavelength of the peak emission, while at the same time displaying a warmer dust temperature than is observed in the

\footnotetext{
11 The high optical depth in Arp 220 characteristically suppresses the emission at short wavelengths, reducing the observable emission from the hot dust component (e.g., Scoville et al. 2017b).
}

lower-redshift ALESS sources (Swinbank et al. 2014). The SED of CRLE appears to closely resemble that of GN20, an extended $z \sim 4$ DSFG (Figure 5; e.g., Carilli et al. 2010; Magdis et al. 2011; Hodge et al. 2012, 2015). Our reference SED templates suggest a potential redshift trend toward hotter dust in higher-redshift DSFGs, as evidenced by the shorter-wavelength SED peak in CRLE relative to the ALESS sample average (mostly in the range $z \sim 2-3$; Danielson et al. 2017) and the longer wavelength in comparison to HFLS3 $(z=6.34$; Riechers et al. 2013; Faisst et al. 2017). Heating from a warmer cosmic microwave background $(\mathrm{CMB})$ may partly contribute to this tentative dust temperature trend (e.g., da Cunha et al. 2013). However, selection effects may also be partly responsible for this trend, since most of these DSFGs were selected at fixed observed-frame wavelengths.

\subsection{Radio Continuum Emission}

CRLE is detected at $7 \sigma$ significance in $3 \mathrm{GHz}$ continuum emission (24.3 $\pm 3.8 \mu \mathrm{Jy}$; Smolčić et al. 2017b). The emission 

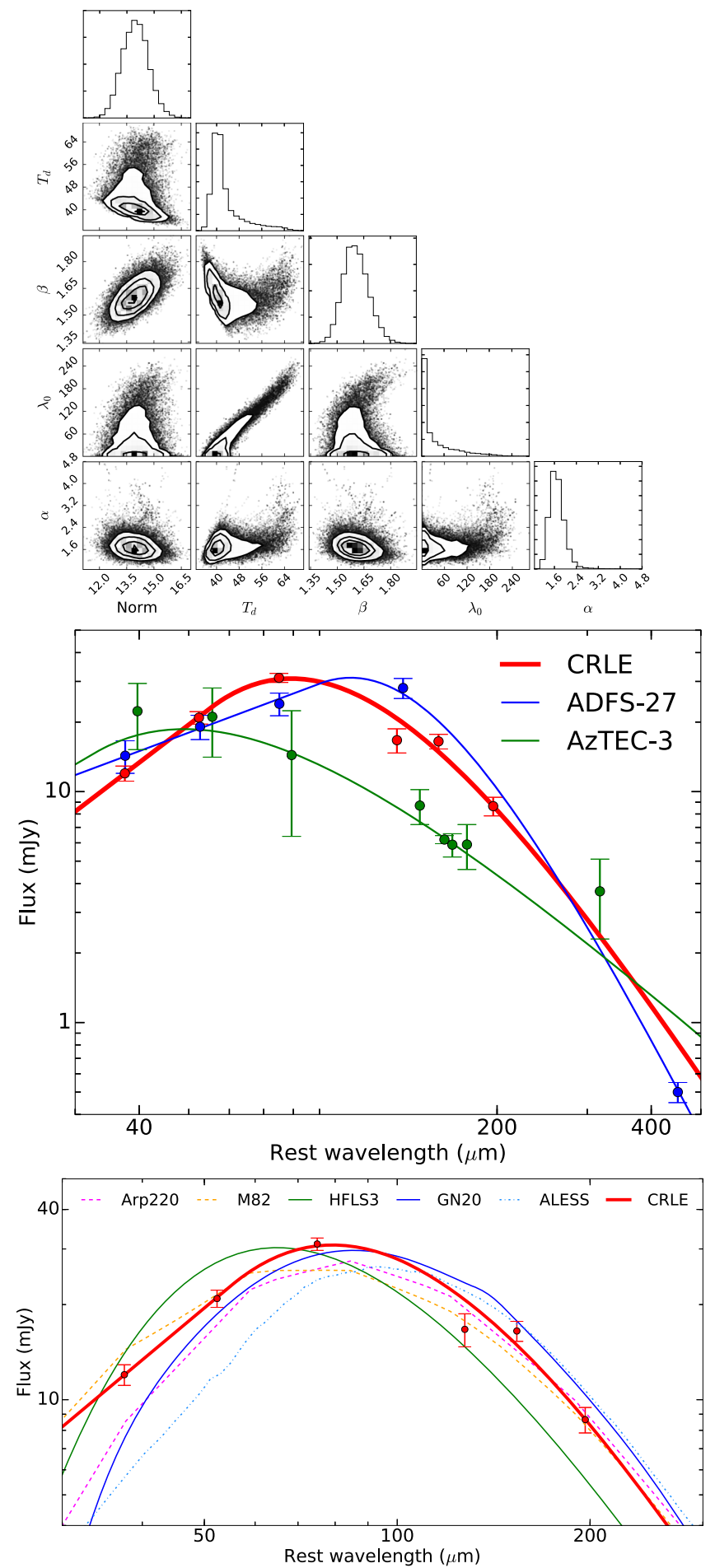

Figure 5. Modified blackbody fits to the FIR SED in CRLE. Top: corner plot of the model parameter posterior distribution. Middle: FIR SED comparison to other $z>5$ DSFGs (AzTEC-3 and ADFS-27; Riechers et al. 2014a, 2017). CRLE shows peak emission at intermediate rest wavelengths between the hot compact starburst AzTEC-3 and the early-stage merger ADFS-27. Bottom: comparison to other low- and high-redshift FIR SED templates (Silva et al. 1998; Magdis et al. 2011; Riechers et al. 2013; Swinbank et al. 2014).

is not resolved at a synthesized beam size of 0.75 , and it is aligned with the dust continuum emission (Figure 4). CRLE is also potentially weakly detected at $1.4 \mathrm{GHz}$ at the $2.5 \sigma$ level (Schinnerer et al. 2010). We conservatively adopt a $3 \sigma$ limit of
Table 3

Results from Modified Blackbody Fitting to the FIR SED of CRLE

\begin{tabular}{lccccc}
\hline \hline Percentile & $\begin{array}{c}\text { Norm. }(158 \mu \mathrm{m}) \\
(\mathrm{mJy})\end{array}$ & $\begin{array}{c}T_{d} \\
(\mathrm{~K})\end{array}$ & $\beta$ & $\begin{array}{c}\text { Rest } \lambda_{0} \\
(\mu \mathrm{m})\end{array}$ & $\alpha$ \\
\hline 16th & 13.25 & 38.95 & 1.537 & 1.659 & 1.430 \\
50th & 13.94 & 41.16 & 1.604 & 15.77 & 1.658 \\
84th & 14.65 & 47.49 & 1.674 & 80.08 & 1.938 \\
\hline
\end{tabular}

$<84 \mu \mathrm{Jy}$ at $1.4 \mathrm{GHz}$, which is consistent with a spectral index of -0.7 . Sensitive low-radio-frequency observations also provide constraining nondetections (K. Tisanić et al. 2018, in preparation). We derive $3 \sigma$ upper limits of $320 \mu \mathrm{Jy}$ at $325 \mathrm{MHz}$ and $150 \mu \mathrm{Jy}$ at $610 \mathrm{MHz}$. These imply constraints to the effective radio spectral index to the observed-frame $3 \mathrm{GHz}$ of $>$ -1.16 and $>-1.14$ for the 325 and $610 \mathrm{MHz}$ limits, respectively.

Adopting the redshift-dependent FIR-radio correlation measured by Delhaize et al. (2017), we can convert our measured $3 \mathrm{GHz}$ flux and the constraints to the $1.4 \mathrm{GHz}$ flux to FIR luminosities. The FIR-radio correlation has been calibrated at a rest-frame frequency of $1.4 \mathrm{GHz}$, which corresponds to observed-frame $210 \mathrm{MHz}$ for CRLE. This comparison therefore requires significant extrapolation from our measurement at $3 \mathrm{GHz}$, and it is sensitive to the spectral index. Adopting a radio power-law index of -0.7 , as in Delhaize et al. (2017), we use $3 \mathrm{GHz}$ flux and a $1.4 \mathrm{GHz}$ flux upper limit to derive FIR luminosities of $4.0 \times 10^{12}$ and $<8.0 \times 10^{12} L_{\odot}$, respectively. This radio-inferred FIR luminosity is significantly lower than our direct measurement. We could reconcile these estimates with an effective spectral index of -1.2 between restframe 20 and $1.4 \mathrm{GHz}$, but this would be in slight tension with our 325 and $610 \mathrm{MHz}$ upper limits. Alternatively, Molnár et al. (2018) suggested that the FIR-radio correlation may not evolve in star-forming, disk-dominated galaxies. If we assume no redshift evolution, the $3 \mathrm{GHz}$ flux and $1.4 \mathrm{GHz}$ flux upper limit would imply FIR luminosities of $\sim 2.7 \times 10^{13}$ and $<5.5 \times 10^{13} L_{\odot}$, in agreement with our direct measurement.

However, the observed radio emission may not be well described by a single power-law spectral index down to restframe $1.4 \mathrm{GHz}$. In particular, the analysis by Tabatabaei et al. (2017) suggests that approximately half of the radio flux at this frequency may be due to thermal free-free emission. Under this assumption, and adopting the relationship between thermal radio emission and SFR by Murphy et al. (2011) for an H II region electron temperature of $T_{e}=10^{4} \mathrm{~K}$, we would infer an $\mathrm{SFR} \sim 4000 M_{\odot} \mathrm{yr}^{-1}$ from the $3 \mathrm{GHz}$ continuum flux, with large uncertainties due to assuming a thermal versus nonthermal fraction.

\subsection{Star-forming Gas Properties}

Low- $J$ CO line luminosities are expected to provide a reliable estimate of the molecular gas mass (e.g., Bolatto et al. 2013). Here we assume a brightness temperature ratio of $R_{21}=1$ between the CO $J=2-1$ and $1-0$ lines, due to the moderately high inferred dust temperature of CRLE (Table 3). ${ }^{12}$ Another effect that may be relevant to the interpretation of the observed $\mathrm{CO}$ line flux is contrast against the warmer $\mathrm{CMB}$ and the

\footnotetext{
12 Previous samples of DSFGs at lower redshift show nearly thermalized gas excitation up to the $J=2-1$ transition, justifying our assumption $\left(R_{21} \sim 0.85-0.95\right.$; e.g., Carilli \& Walter 2013).
} 
additional gas heating this provides at $z>5$ (da Cunha et al. 2013). While the effect this may introduce is difficult to estimate without additional $\mathrm{CO}$ excitation constraints, da Cunha et al. (2013) suggested that for warm gas kinetic temperatures and moderately high gas densities, we may expect the observed $\mathrm{CO}$ line flux to be suppressed by $\sim 0.5-0.8$ at this redshift. We do not attempt to estimate a correction factor for this effect but include it in the definition of $\alpha_{\mathrm{CO}}$ instead (i.e., a larger CMB correction would imply a higher $\alpha_{\mathrm{CO}}$ ). By assuming the dynamical mass to be completely accounted for by molecular gas, we can derive a conservative upper limit on the $\mathrm{CO}$ conversion factor of $\alpha_{\mathrm{CO}}<2.1 \pm 0.6 M_{\odot}\left(\mathrm{K} \mathrm{km} \mathrm{s}^{-1} \mathrm{pc}^{2}\right)^{-1}$. This conversion factor is conservative, because it does not include the stellar contribution to the dynamical mass. Our stellar mass estimate is too uncertain to provide a useful constraint in this context. Such low conversion factors are typically only observed in highly metal-enriched galaxies, which appears to suggest that metallicity is not a major source of uncertainty in the interpretation of line ratios (Bolatto et al. 2013). Furthermore, from the dust mass estimates presented in Section 4.2, we can adopt a gas-to-dust ratio in order to derive an independent estimate of $\alpha_{\mathrm{CO}}$. The gas-to-dust ratio may be assumed to follow the same dependence on metallicity as measured at lower redshift. Here we follow Magdis et al. (2011) by adopting a super-solar metallicity, expressed as $12+$ $\log (\mathrm{O} / \mathrm{H})$, between 8.8 and 9.2. This implies a gas-to-dust mass ratio of 35-75, which is consistent with an average value for DSFGs of $\sim 50$ (Santini et al. 2010). This range implies a range of $\alpha_{\mathrm{CO}}$ from $0.65 \pm 0.16$ to $1.4 \pm 0.3 M_{\odot}\left(\mathrm{K} \mathrm{km} \mathrm{s}^{-1} \mathrm{pc}^{2}\right)^{-1}$. These ranges are consistent with expectations from local ULIRGs, for which a conversion factor of $\alpha_{\mathrm{CO}} \sim 0.8 M_{\odot}\left(\mathrm{K} \mathrm{km} \mathrm{s}^{-1} \mathrm{pc}^{2}\right)^{-1}$ was estimated (Downes \& Solomon 1998). In the following, we adopt a conversion factor of $\alpha_{\mathrm{CO}}=1 M_{\odot}\left(\mathrm{K} \mathrm{km} \mathrm{s}^{-1} \mathrm{pc}^{2}\right)^{-1}$, which provides a conservative estimate of the molecular gas mass in CRLE. ${ }^{13}$ We thus derive a total molecular gas mass of $M_{\text {gas }}=(7.0 \pm 0.5) \times 10^{10} M_{\odot}$, corresponding to $\sim 50 \% \pm 15 \%$ of the dynamical mass estimate. This gas mass estimate is similar to the gas masses of other $z>5$ DSFGs, such as AzTEC-3 $\left(\sim 5.3 \times 10^{10} M_{\odot}\right)$ and HFLS3 $\left(\sim 4.5 \times 10^{10} M_{\odot}\right.$; Riechers et al. 2010, 2013; Cooray et al. 2014). Our inferred $\alpha_{\mathrm{CO}}$ is compatible with model predictions by Vallini et al. (2018) at $z \sim 6$, which suggest $\alpha_{\mathrm{CO}}=(1.5 \pm 0.9) M_{\odot}\left(\mathrm{K} \mathrm{km} \mathrm{s}^{-1} \mathrm{pc}^{2}\right)^{-1}$, despite the subsolar metallicity of their simulated galaxy. They interpret this low conversion factor as the result of the high density and turbulence in the molecular gas of their simulated galaxy. The measured $[\mathrm{C} \mathrm{II}] / \mathrm{CO}(2-1)$ line ratio in CRLE is also compatible with their model predictions, although we caution that their simulated galaxy targets the "normal" star-forming population with an SFR over an order of magnitude lower than CRLE (Vallini et al. 2018).

We also use single-wavelength dust continuum emission measurements on the Rayleigh-Jeans tail to explore alternative methods to estimate the ISM mass of CRLE. Using the the restframe $205 \mu \mathrm{m}$ and $1.3 \mathrm{~mm}$ observations, we follow Scoville et al. $(2016,2017 \mathrm{a})$ to estimate total ISM masses of $1.1 \times 10^{12}$ and $8.0 \times 10^{11} M_{\odot}$, respectively. This method was calibrated at a rest-frame wavelength of $850 \mu \mathrm{m}$; hence, the latter estimate is expected to be more reliable, since the

\footnotetext{
${ }^{13}$ We note that there remain substantial systematic uncertainties regarding the appropriate CO conversion factor for high-redshift DSFGs, as well as for local ULIRGs (e.g., Scoville et al. 2016, 2017b). Previous assumptions of $\alpha_{\mathrm{CO}}$ may also affect the adopted gas-to-dust ratios and may therefore be partly responsible for the apparent consistency of our estimates.
}

extrapolation is smaller. We also use rest-frame 500 and $250 \mu \mathrm{m}$ fluxes in CRLE to infer a gas mass following Groves et al. (2015). The rest-frame 500 and $250 \mu \mathrm{m}$ fluxes are estimated from our best-fitting modified blackbody model and imply gas masses of $1.3 \times 10^{12}$ and $2.4 \times 10^{11} M_{\odot}$, respectively. Therefore, these dust-based methods would imply very large ISM masses, exceeding our dynamical mass estimate and suggesting that hotter dust temperatures may affect these techniques, making them less reliable for this type of highredshift galaxy (e.g., Scoville et al. 2015).

Adopting the relationship between CO luminosity and SFR (i.e., the star formation law) for local starburst galaxies (Kennicutt 1998; Carilli \& Walter 2013), we can convert the measured $L_{\text {CO }}^{\prime}$ to an IR luminosity of $L_{\mathrm{IR}}=1.3 \times 10^{13} L_{\odot}$ with large uncertainties (the scatter of this relationship is a factor of 2.5 ). This is consistent with our measured IR luminosity, although apparently at the low end of the confidence interval. By adopting the fiducial values for the SFR and the molecular gas mass, we derive a gas depletion timescale of $45 \pm 4 \mathrm{Myr}$, which is comparable to estimates for other $z>5$ DSFGs, such as AzTEC-3 ( 50 Myr) and HFLS3 ( 36 Myr) (Riechers et al. 2010, 2013, 2014a).

\subsection{Merger Stage of CRLE}

As shown in Section 4.2, the dust SED in CRLE appears to be intermediate between those of other $z>5$ DSFGs, such as AzTEC-3 and ADFS-27 (Figure 5; Riechers et al. 2010, 2014a, 2017). A possible interpretation of these differences in the dust properties (e.g., temperature or optical depth) among these galaxies involves the merger stage. In particular, AzTEC-3 appears to be a dispersion-dominated starburst with an exceptionally compact central density profile that is suggestive of a late-stage merger after coalescence (D. Riechers et al. 2018, in preparation). On the other hand, ADFS-27 is an interacting galaxy pair, where the individual galaxies are separated by just $9 \mathrm{kpc}$ and therefore may represent an early merger stage (Riechers et al. 2017). In Appendix C, we carry out dynamical modeling of the [C II] emission, which suggests that a simple rotating disk model does not provide a good fit to CRLE. This conclusion is also supported by the asymmetric profile visible in the line spectra (Figure 2). We find evidence for a second dynamical component, which may suggest an overall dispersion-dominated system. This evidence appears to suggest an ongoing merger in CRLE with a component separation of $2.0 \pm 0.4 \mathrm{kpc}$ (from the [C II] emission modeling). Therefore, we tentatively interpret the intermediate SED in CRLE as a reflection of its merger stage. However, the SED in CRLE also resembles that in GN20, which shows a coherently rotating massive gas disk and no evidence for a galaxy merger (Hodge et al. 2012).

\section{Galaxy Overdensity Around CRLE}

\subsection{Overdensity Characterization}

The close association in the sky and redshift space to HZ10 $\left(z=5.654,13^{\prime \prime}\right.$ distance, corresponding to only $77 \mathrm{kpc}$ in projection and $\sim 580 \mathrm{~km} \mathrm{~s}^{-1}$ of relative radial velocity) suggests that CRLE may be located in an overdense galaxy environment. We follow the procedure described in Smolčić et al. (2017a) to evaluate a potential galaxy overdensity around CRLE. First, we analyze the small-scale overdensity around the DSFG in the COSMOS2015 catalog, making use of the 

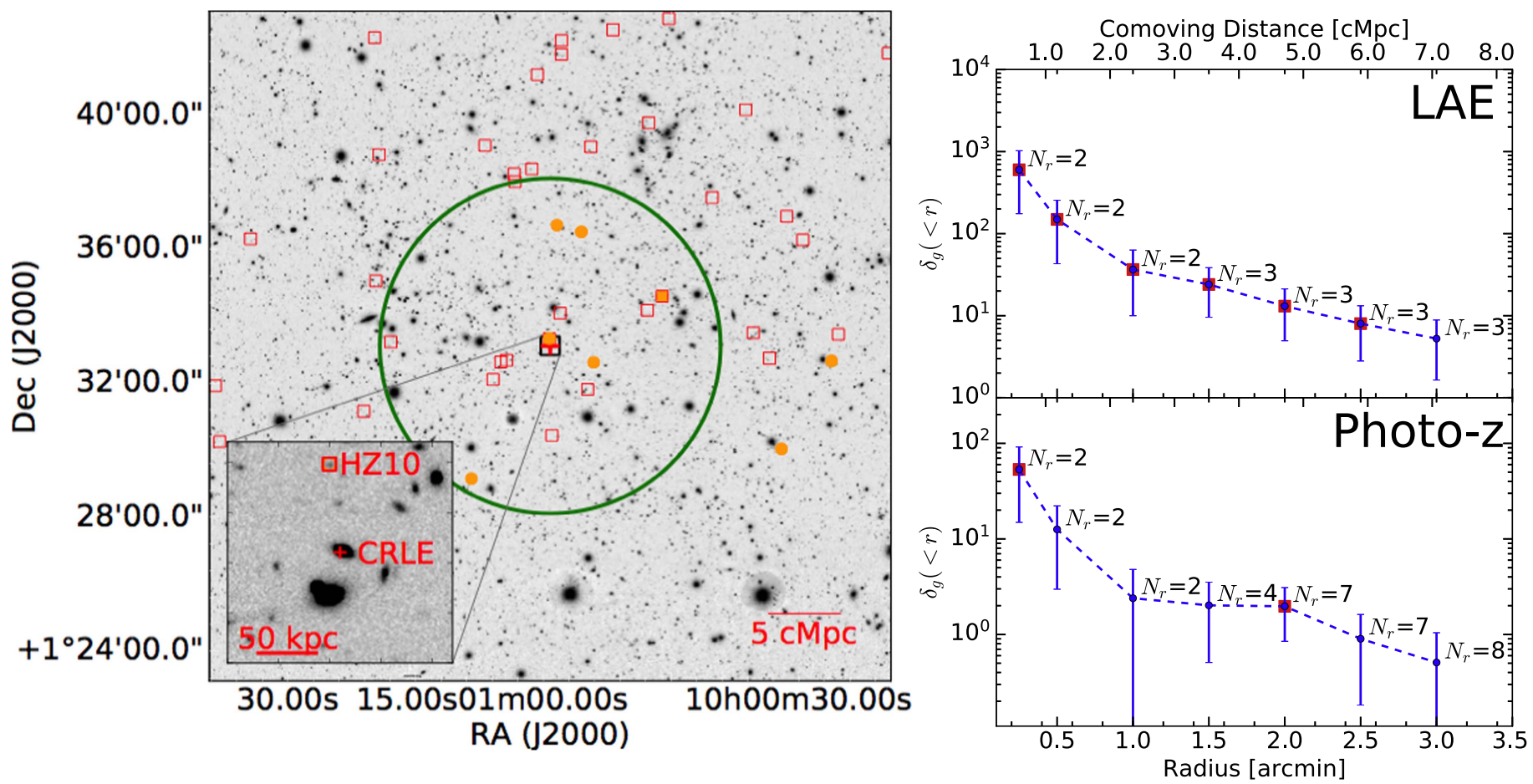

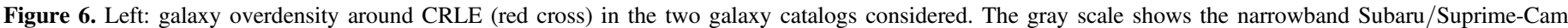

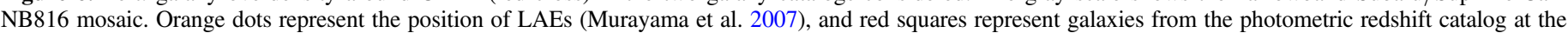

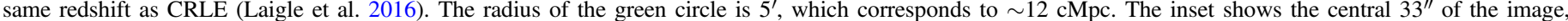

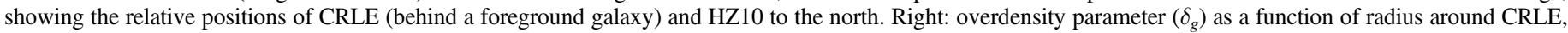

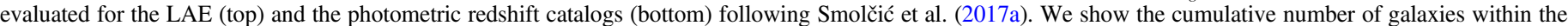
specified radius. Red squares indicate a significant overdensity, with a false-positive rate below $5 \%$.

photometric redshift information, as detailed in Smolčić et al. (2017a). Then, we apply a similar technique to also investigate the galaxy overdensity in the LAE catalog at $z \sim 5.7$, which may offer a higher accuracy in the redshift range selected (Murayama et al. 2007).

We do not apply the magnitude cut $i^{+}<25.5$ of Smolčić et al. (2017a), because the $i^{+}$magnitude of HZ10 is 26.45 , which we know to have a redshift in the correct range. Based on the magnitude of HZ10, we also expect that even massive galaxies at this high redshift are likely to have an $i^{+}$magnitude below the adopted threshold of Smolčić et al. (2017a). On the other hand, our choice to include fainter galaxies may limit the photometric redshift accuracy. We adopt their choice of redshift binning, which implies a photo- $z$ range of $\Delta z=0.64$ for the catalog "slice." Following Smolčić et al. (2017a), we define a galaxy overdensity parameter by $\delta_{g}(r)=\frac{N_{r}}{\Sigma_{\mathrm{bg}} \pi r^{2}}-1$, where $N_{r}$ is the number of galaxies in the catalog within a radius $r$ of the DSFG (including the DSFG itself), and $\Sigma_{\mathrm{bg}}$ is the mean galaxy surface density in the redshift "slice." We take into account masked regions when evaluating the surface area. We evaluate the overdensity parameter around CRLE at radii of 0 .25 (to capture the overdensity due to HZ10 alone) and $0.5-3^{\prime}$ in steps of 0.5 in both the photometric redshift and LAE catalogs (Figure 6).

In order to evaluate the significance of the measured overdensity parameter values, we follow the procedure by Smolčić et al. (2017a), producing 10 mock random catalogs adopting the same masked regions with the same number of galaxies as the real ones and measuring the overdensity around 1000 randomly placed centers. The rate of chance occurrence of the actual $N_{r}$ profile (equivalently, of the overdensity parameter) can then be evaluated. We mark significantly overdense radii with squares in Figure 6, adopting the same 5\% false-positive rate as Smolčić et al. (2017a).

In the COSMOS2015 photometric redshift catalog, we find that HZ10, due to its very close separation, constitutes a significant overdensity of $>50$, and that at a radius of $2^{\prime}$, there is only a $1 \%$ probability of the observed overdensity (seven galaxies; corresponding to $\delta_{g} \sim 2-3$ ) being produced by chance. The chance probability of at least one neighbor within 0.5 is only $12 \%$ (the nearest, HZ10, is at 0.216 ). This chance probability is comparable to that of having at least two neighbors within 1!05 (i.e., the separation to the next galaxy) or at least three neighbors within 1!5 (the following galaxy). In the LAE catalog, the overdensity is even more significant, due to the lower contamination from galaxies at incorrect redshifts. We find a significant overdensity at all radii up to 2.5 , with HZ10 already implying an overdensity of $>500$ and a second LAE within 1.5 constituting an overdensity of $\sim 30$. The overdensity in the LAE catalog at a radius of $5^{\prime}$, including a total of seven galaxies, is also $>4$.

We also explore the second technique utilized by Smolčić et al. (2017a) in order to study the larger-scale overdensity. However, we are limited to scales of $4^{\prime}-5^{\prime}$ in the LAE catalog and $3^{\prime}$ in the photometric redshift catalog, since CRLE is close to the southern edge of the catalog. This method utilizes a Voronoi tessellation analysis (VTA) in order to identify overdense regions and then determines an overdensity center by evaluating a barycenter for the "region." It then evaluates the significance of the overdensity parameter value as a function of radius around this newly found center. The VTA 
analysis in the case of CRLE indicates that, in both the photometric redshift and LAE catalogs, CRLE and HZ10 are in an overdense region (i.e., above the 80th percentile of a randomized galaxy density distribution) due to the close proximity to the next galaxy neighbors. The overdensity center is evaluated to be approximately the midpoint between CRLE and HZ10. Because the overdensity center is close to CRLE, the overdensity evaluation for this method reproduces similar results to those of our previous analysis.

Four galaxies (all part of the LAE catalog) within $5^{\prime}$ have a spectroscopic redshift that place them in the overdensity (5.665, 5.674, 5.688, and HZ10 at 5.659 from Keck/DEIMOS; P. Capak et al. 2018, in preparation). The other two LAEs within this radius have no known spectroscopic redshifts. ${ }^{14}$ Of the four LAEs with spectroscopic redshift confirmation, two (including HZ10) are also part of the photometric redshift sample. No other photometric redshift candidates within $5^{\prime}$ have a spectroscopic redshift. If we expand the search radius to $17^{\prime}$, corresponding to $40 \mathrm{cMpc}$ in the spectroscopic redshift catalog, we find four more galaxies with spectroscopic redshifts between 5.5 and 6 , which may potentially be part of the same overdensity (with spectroscopic redshifts of 5.682, 5.728, 5.663, and 5.742).

\subsection{Discussion of the Overdensity Significance}

Here we compare the galaxy overdensity around CRLE, with the cases of AzTEC-3 and other lower-redshift DSFGs (e.g., Walter et al. 2012; Oteo et al. 2018; Smolčić et al. 2017a), with LAE overdensities at $z>5$ (Higuchi et al. 2018) and in relation to theoretical expectations (e.g., Chiang et al. 2013, 2017).

The closest analog to the galaxy overdensity around CRLE is the protocluster around AzTEC-3 at $z=5.3$, which is also located in the COSMOS field (Riechers et al. 2010, 2014a; Capak et al. 2011). The close proximity of the "normal" LAE/ LBG HZ10 to CRLE ( $\sim 77 \mathrm{kpc}$ away) is comparable to the close separation between the luminous DSFG AzTEC-3 and the "normal" galaxy LBG-1 ( $95 \mathrm{kpc}$ away; Riechers et al. 2014a). Capak et al. (2011) reported a rich galaxy overdensity around AzTEC-3 with an overdensity parameter of $\gtrsim 11$ within a $2 \mathrm{cMpc}$ radius. We also detect a comparable overdensity of LAEs of $\delta_{g} \gtrsim 10$ within a radius of $\sim 5 \mathrm{cMpc}$ of CRLE. Because of the higher redshift of CRLE $(z \sim 5.667)$ than AzTEC-3 $(z \sim 5.298)$, the catalogs contain significantly fewer galaxies. The overdensity is therefore associated with a smaller number of galaxies and hence is subject to larger uncertainties from random fluctuations. The "normal," "companion" galaxies LBG-1 and HZ10 are similar in terms of their stellar masses and UV luminosities, but the FIR luminosity in HZ10 is almost an order of magnitude higher, suggesting that the ISM in HZ10 may by substantially more enriched (Capak et al. 2015; Pavesi et al. 2016). Furthermore, the [C II]/[N II] ratio appears to suggest that the metallicity of HZ10 is compatible with local and high-redshift starburst galaxies, while it suggests a lower metallicity for LBG-1 (Pavesi et al. 2016). The overdense environment experienced by HZ10 may be partly responsible for the particularly enriched ISM state in this "normal" galaxy. In particular, it is possible that metal-enriched material that was ejected by CRLE may have been accreted by

\footnotetext{
14 One of them, the closest LAE after HZ10, has an incorrect photometric redshift of 0.7688 ; the other one is not in the photometric catalog.
}

HZ10. Also, it is likely that the local dark matter overdensity, as suggested by the galaxy overdensity, may be responsible for the early galaxy growth by providing abundant gas fueling to the central regions. However, LBG-1 seems to be located in a similar environment and does not display a comparable level of enrichment. Capak et al. (2011) estimated that the dynamical time for LBG-1 to reach AzTEC-3 may be of order $\sim 0.5 \mathrm{Gyr}$, providing several dynamical times for a merger to occur by $z \sim 2$. A similar estimate applies to HZ10 and its likely eventual merger with CRLE, which may produce a central cluster galaxy.

Smolčić et al. (2017a) analyzed galaxy overdensities around previously known DSFGs in the COSMOS field at $z \sim 1-5.3$ and found an incidence of approximately $\sim 50 \%$ when using methods similar to the ones employed here. They tentatively found a higher occurrence of DSFGs occupying overdense environments at $z>3$ than at $z<3$. This would be compatible with our finding of a high galaxy overdensity including CRLE at $z \sim 5.7$. A higher incidence of overdensities associated with the highest-redshift DSFGs would be consistent with the idea that these massive galaxies may be associated with the highest peaks of the density field, tracing the most massive dark matter halos at early cosmic epochs (e.g., Springel et al. 2005; Li et al. 2007; Overzier et al. 2009; Capak et al. 2011).

Recent observations with the Hyper-Suprime-Cam on the Subaru telescope have yielded a deeper and wider catalog of LAEs in COSMOS at $z \sim 5.7$ than available for our analysis (Ouchi et al. 2018; Shibuya et al. 2018). These authors reported numerous LAE overdensities at this redshift, showing that a significant fraction of star-forming galaxies at this epoch may be part of protocluster environments (Higuchi et al. 2018). In particular, these authors report an overdensity, HSC-z6PCC5, in the COSMOS field, with an overdensity parameter of $\delta \sim 10$. The reported overdensity center is located $44 \mathrm{cMpc}$ away from CRLE and at a redshift of $z=5.686$, corresponding to only $9 \mathrm{cMpc}$ of radial separation. It is therefore possible that CRLE and its associated small-scale galaxy overdensity may also be associated with this significantly larger-scale early cosmic structure.

Recent theoretical work suggests that protoclusters may have dominated star formation in the first 2 billion yr of cosmic time (Chiang et al. 2013, 2017). This is due to the fact that the fraction of the cosmic volume occupied by all future (proto) clusters increases by nearly three orders of magnitude from $z=0$ to 7 . More importantly, most models suggest that early galaxy formation may be dominated by the central regions of the most massive overdensities and that star formation may evolve inside-out to galaxies in lower-density environments (Chiang et al. 2017). These may be crucial predictions of structure formation in the early universe. A quantification of the fraction of star formation in different environments as a function of cosmic time may be an important cosmological probe in the era of wide-area surveys. The physical processes associated with the "central" galaxy forming in a protocluster, which may be a DSFG at least during part of its life, may strongly affect the evolution of such protoclusters, both by enriching the intracluster medium and by providing energy input through winds and radiation.

\section{Conclusions}

We have reported the serendipitous discovery of the bright, dusty, starbursting galaxy known as CRLE at $z=5.667$ in the 
first billion years of cosmic time. This galaxy represents the highest-redshift and brightest DSFG in the COSMOS field known to date, providing a higher redshift and brighter analog to the $z=5.3$ massive starburst AzTEC-3. We report the detection of [C II], [N II], and $\mathrm{CO}(2-1)$ line emission, and we find properties that are common among the highest-redshift DSFGs. CRLE displays a large molecular gas reservoir $\left(\sim 7 \times 10^{10} M_{\odot}\right)$, a short gas depletion timescale of order $\sim 50 \mathrm{Myr}$ characterizing the intense starburst, and a highintensity radiation field, as evidenced by a deep [C II] deficit. We find evidence for a significant fraction of the [C II] emission to be coming from ionized gas, similar to other high-redshift DSFGs. We suggest that this emission may be coming from a diffuse ionized medium not directly associated with the dense star-forming gas. We find dynamical evidence and dust emission properties consistent with an intermediate-stage merger. The physical proximity of the previously known "normal" LAE HZ10 to CRLE constitutes a high overdensity and suggests that these two galaxies may coalesce in the future, forming a massive central cluster galaxy. We find further evidence for a galaxy overdensity using both photometric redshift and LAE catalogs, which indicates the location of a likely protocluster analogous to the case of AzTEC-3. The presence of this likely protocluster supports the idea that such bright, extremely early starburst galaxies may commonly be associated with the most massive dark matter halos in the universe at their respective epochs, providing the earliest sites of star formation of the most massive central cluster galaxies that we observe in the local universe.

We thank the anonymous referee for a helpful and constructive report. R.P. and D.R. acknowledge support from the National Science Foundation under grant number AST1614213 to Cornell University. R.P. acknowledges support through award SOSPA3-008 from the NRAO. The National Radio Astronomy Observatory is a facility of the National Science Foundation operated under cooperative agreement by Associated Universities, Inc. This paper makes use of the following ALMA data: ADS/JAO.ALMA\#2015.1.00928.S, 2015.1.00388.S, 2012.1.00523.S. ALMA is a partnership of ESO (representing its member states), NSF (USA), and NINS (Japan), together with NRC (Canada), MOST and ASIAA (Taiwan), and KASI (Republic of Korea), in cooperation with the Republic of Chile. The Joint ALMA Observatory is operated by ESO, AUI/NRAO, and NAOJ.

\section{Appendix A \\ Constraining the Effects of Gravitational Lensing}

The high apparent luminosity of CRLE, combined with the coincident spatial position with a foreground galaxy, raise questions concerning the relative importance of strong gravitational lensing. About $10 \%$ of the area of the segmentation map in the HST $H$-band images we utilized to study CRLE is occupied by (mostly) local galaxies. Therefore, the coincidence of galaxies at different redshifts may not be uncommon. Using the method of Harris et al. (2012), we roughly estimate the magnification due to gravitational lensing based on the apparent correlation between intrinsic CO luminosity and line width, finding $\mu=0.9 \pm 0.2$. This may suggest that lensing is not expected to significantly boost the observed luminosity of CRLE. However, we note that the Harris et al. (2012) method relies on a proposed $L_{\mathrm{CO}}^{\prime}-\mathrm{FWHM}_{\mathrm{CO}}$ relation, which may only hold with large scatter (e.g., Sharon et al. 2016).

We also explore the potential lensing magnification by modeling the foreground galaxy with the commonly used approximation of a singular isothermal sphere (SIS). We confirm that the light distribution appears to be approximately proportional to the aperture radius, which implies that a "lighttraces-mass" model would be approximately equivalent to the adopted SIS. By measuring the flux in the HST/WFC3 F160W image in apertures of varying radius, we deduce that $68 \%$ of the light is included within $1^{\prime \prime}$ radius, $37 \%$ within 0.15 radius, and $22 \%$ within $0 . " 3$ radius. We assume that the total mass in the central regions of the foreground galaxy should be dominated by the stellar mass. Therefore, we adopt a total mass of $3.3 \times 10^{9} M_{\odot}$, as reported by the COSMOS2015 catalog (Laigle et al. 2016), in agreement with our Cigale SED modeling. A combination of the distance implied by the photometric redshift, the measured size, and the total mass suggests a velocity dispersion parameter for the SIS model of only $25-30 \mathrm{~km} \mathrm{~s}^{-1}$. This velocity dispersion parameter corresponds to a very small Einstein radius of $\sim 0$ ". $02-0$ ". 025 at the lens and source distances derived by their redshifts. We measure a separation of the DSFG from the lens center of $\sim 0$ ". 3 . If we were to assume a point-source model for the DSFG, we would estimate a magnification of $<10 \%$. The relative positional uncertainty is dominated by the HST astrometric uncertainty, which we assume to be $\sim 0$." 1 (e.g., Gómez-Guijarro et al. 2018), dominating over the ALMA positional accuracy or the fitting uncertainty.

We use both our custom code and the publicly available code gravlens (Keeton 2001) to constrain the effect of lensing in a model source distribution, obtaining equivalent answers. We fix the lens Einstein radius to 0 !" 025 , the source spatial FWHM to $0 . " 6$ based on the measured [C II] size, and a mean lenssource separation of 0.3 . We randomly vary this positional separation by adding independent, normally distributed spatial offsets along the two axes with a standard deviation of 0 ". 1 , representing the relative positional uncertainty. This results in an approximately normally distributed magnification of $\mu=1.09 \pm 0.02$. Furthermore, since the Einstein radius is so small compared to the spatial extent of the source $(<5 \%)$, the effects of lensing are negligible both to the global flux and to the observed kinematic structure within the uncertainty of our measurements. In addition, we also allow the source size and relative position to vary and verify that a significantly smaller intrinsic source size is incompatible with the observed source size. The small Einstein radius implies that the observed source size can only be reproduced by a comparable intrinsic size, therefore significantly constraining the allowed magnification to the values reported above. We therefore assume that lensing does not measurably affect any of our conclusions based on the luminosity and spatial structure of CRLE.

\section{Appendix B HST Foreground Galaxy Removal}

In order to constrain the rest-frame optical emission from CRLE, we attempt different methods for removing the contamination of the HST/WFC3 NIR images due to the foreground galaxy. First, we attempt to separate the emission from CRLE from that of the foreground galaxy based on a color difference between the two galaxies (Figure 7). The mean 


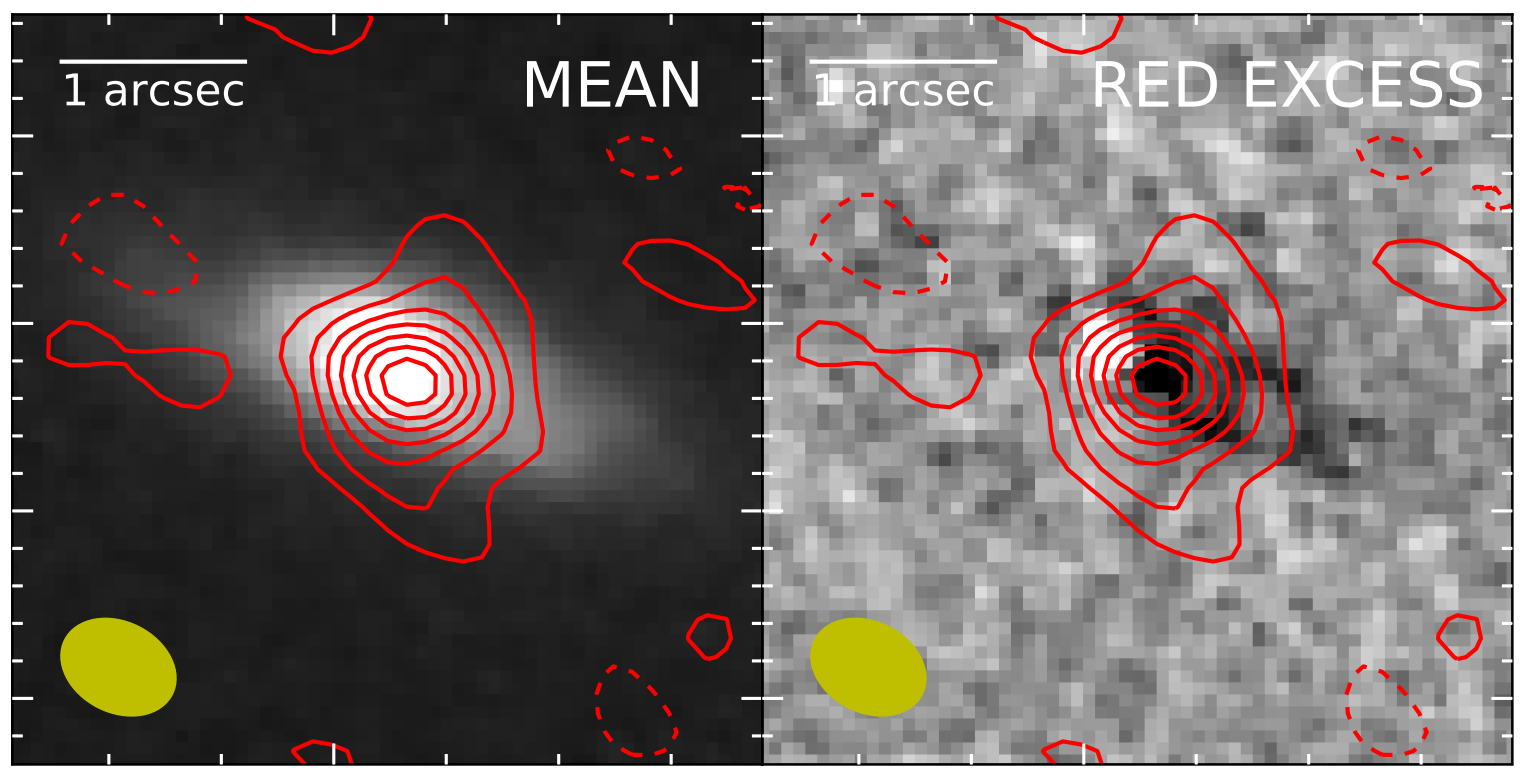

Figure 7. HST NIR-band mean emission and red color excess, attempting to separate the emission from CRLE. Left: ALMA [C II] contours shown in steps of $2 \sigma$, overlaid on the HST NIR mean image. To obtain the mean image, we averaged the emission detected in the three HST/WFC3 bands F105W, F125W, and F160W. Right: difference map of F160W and the mean image, showing F160W emission in excess from the mean.
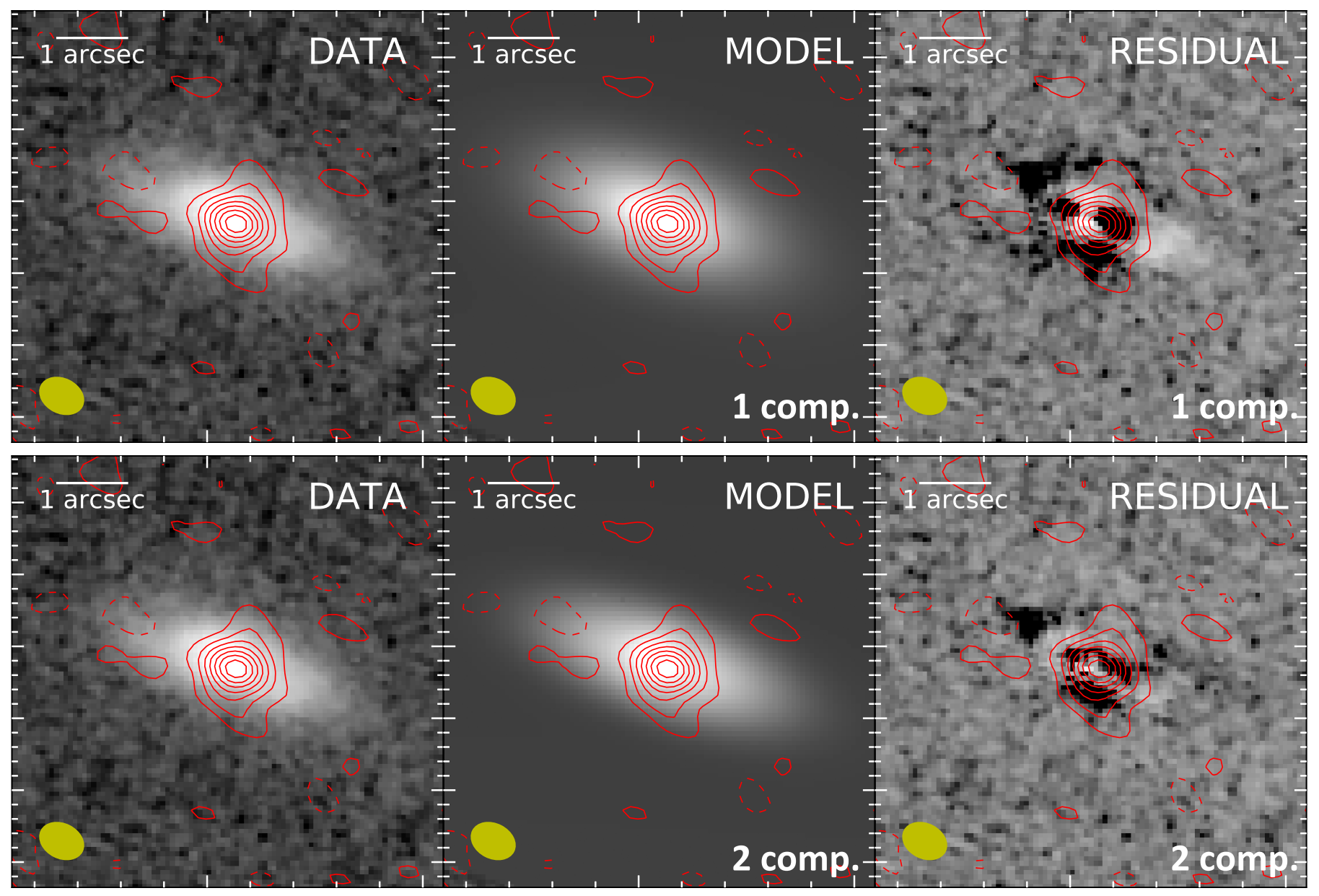

Figure 8. Top: single Sérsic component model fit with Galfit to the HST/WFC3 F160W emission from the foreground galaxy. The low-level residuals show a hint of emission (a few percent in flux) that may be associated with asymmetric structure in the foreground galaxy or CRLE itself (position indicated by the ALMA [C II] contours). Bottom: two-component Galfit best-fit model and residuals, showing that no significant additional structure is present. 
Table 4

Results of One- and Two-component Dynamical Modeling

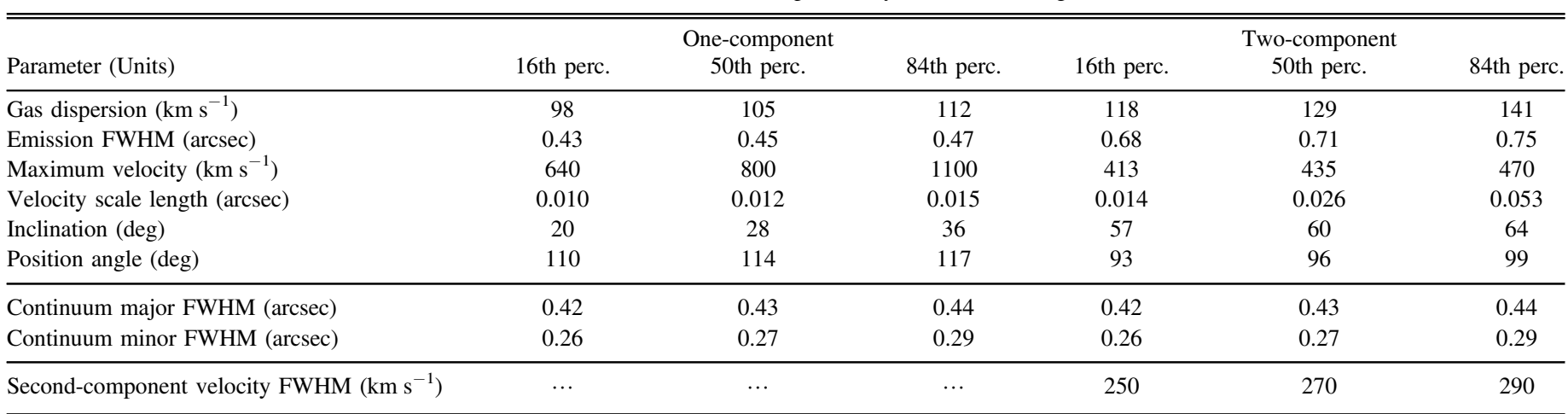

NIR image (average of WFC3/F105W, F125W, and F160W) shows smooth emission due to the foreground disk galaxy, but an F160W "excess" (relative to the mean NIR emission) shows a more red than average component to the northeast of the central position (corresponding to only $\sim 0.5 \%$ of the total F160W flux) and a deficit of F160W emission at the position of the CRLE [C II] emission peak. We may expect the rest-frame optical emission associated with CRLE to appear redder than that of the foreground galaxy. This tentative evidence may therefore suggest that the stellar emission from CRLE may be offset from the [C II] peak as frequently observed in highredshift DSFGs perhaps due to differential dust obscuration or an older stellar population offset from the young massive starforming regions (e.g., Riechers et al. 2014a; Gómez-Guijarro et al. 2018). We also use Galfit to fit Sérsic profile emission models and remove the foreground emission from the F160W image (Peng et al. 2002). We first fit a single-component model characterized by the center position, flux, radius, Sérsic index, axis ratio, and position angle (Figure 8, top). We find a Sérsic index of $\sim 1$, compatible with an exponential disk, and a halflight radius of $0 . " 82$. The total emission is not fit well by this model and shows positive residuals to the northeast of the center, which may or may not be associated with the "red" excess seen in Figure 7. The flux associated with this positive residual is only $\sim 1 \%-2 \%$ of the total F160W flux. Since part of the foreground galaxy emission to the west is apparent in the residuals, we do not consider these residuals to be sufficient to indicate an additional source of emission beyond the imperfect model fit of an intrinsically, not perfectly, symmetric galaxy. In order to achieve a better fit to the foreground emission, we also fit a two-component Sérsic profile (Figure 8, bottom). The residuals do not indicate significant additional structure. The second emission component may be associated with structure in the foreground galaxy or emission from CRLE. In the main text, we neglect the contribution from CRLE to the emission at these wavelengths because it appears to represent, at most, a few percent of the total.

\section{Appendix C Dynamical Modeling}

In order to extract the most precise physical parameters for CRLE, we analyze the [C II] line data, which have the highest signal-to-noise ratio and angular resolution, by dynamical model fitting.
We have developed a novel implementation of dynamical model fitting, working directly on the visibilities. Carrying out the model comparison to data in the UV space, rather than in the image plane, makes our method independent of deconvolution and choice of visibility weighting, and hence more robust. Furthermore, our method takes advantage of the well-behaved (i.e., independent and normally distributed) noise properties of measured visibilities, in comparison to the correlated noise affecting interferometric images.

Our method has general applicability for interferometric data with frequency structure and is based on a Bayesian formulation of the model fitting problem. We use Markov chain Monte Carlo (MCMC) and Nested Sampling techniques in the form of emcee (Foreman-Mackey et al. 2013) and MultiNest for Python (Feroz et al. 2009; Buchner et al. 2014) to sample the posterior distribution for the model parameters and evaluate the model evidence (also called marginal likelihood), i.e., the integral of the posterior that gives the probability of the model given the data. We have verified that the parameter estimates derived from the samples produced by the two different techniques are well within the range of compatibility.

The first model fit to CRLE [C II] is a rotating disk model, generated through the publicly available code KinMSpy (Davis et al. 2013), superposed onto an elliptical 2D Gaussian continuum model. We choose this model to be described by disk center coordinates, systemic velocity, gas dispersion, FWHM size of the spatial light profile of the disk (assumed to be 2D Gaussian), maximum velocity and velocity scale length, inclination, position angle, and line flux. The continuum flux and FWHM sizes of the continuum emission were separately fixed by fitting the line-free channels. We impose nonconstraining priors. We choose uniform priors for additive parameters, logarithmic priors for scale parameters, and a sine prior for the inclination angle. The $1 \sigma$ confidence intervals of the physically relevant parameters derived from our modeling are shown in Table 4, and the median model fit and residuals are shown in Figure 9.

Clear structure is visible in the single-component model residuals by adopting median parameters, particularly in the spectrum, although the total residual flux has formally low significance (Figure 9). We explore a second model with five additional parameters describing a second, unresolved component that is designed to capture the narrow velocity component visible in the spectra and the dispersion map. The additional parameters describe the second-component center $x$ 

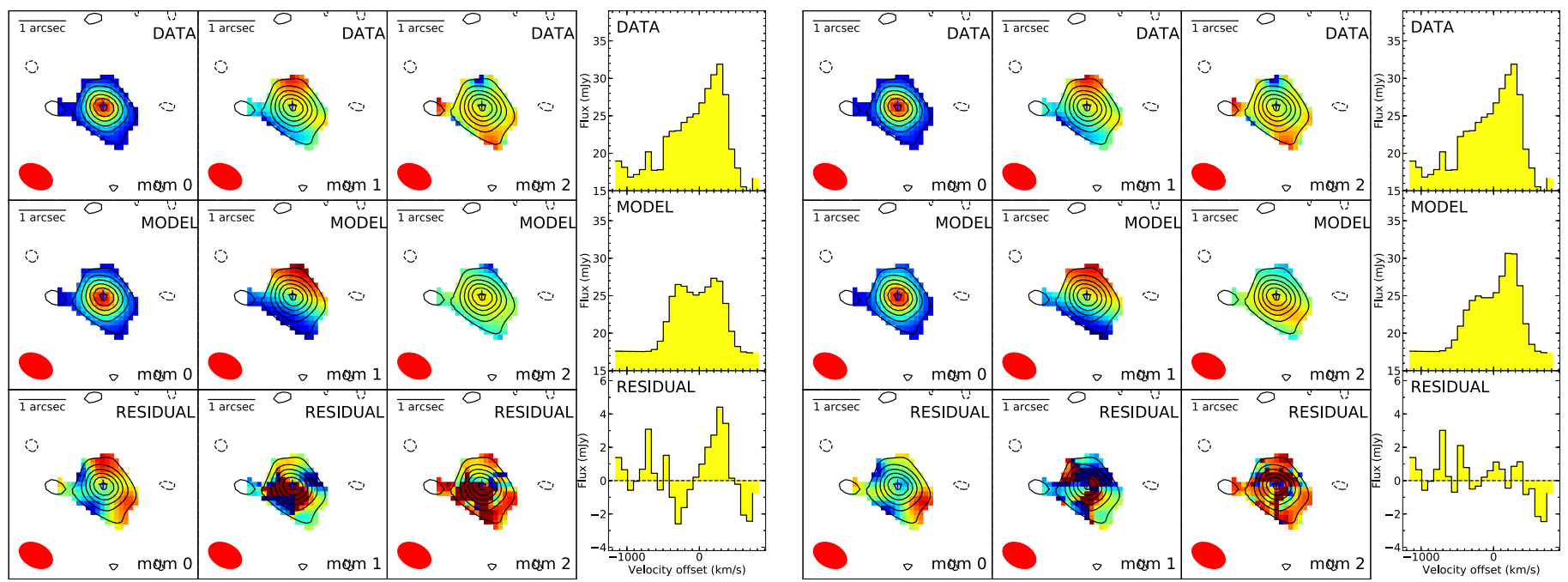

Figure 9. Visibility-space dynamical modeling results for the [C II] line emission in CRLE. We show the "natural" weighting line moment 0 (intensity), 1 (velocity), and 2 (dispersion) maps and spectra for the data, model, and visibility residuals. Left: one-component rotating disk model. Right: two-component model, with a spatially unresolved second dynamical component. Although a one-component model provides an acceptable fit, the residuals show clear spectral structure, and the moment 2 map shows spatial structure hinting at a different dynamical component to the north. The residuals are significantly improved by the addition of a second model component, perhaps suggesting an ongoing galaxy merger.

and $y$ coordinates, systemic velocity, integrated flux, and line velocity width. As Figure 9 shows, the model fit is significantly improved by this additional component. We characterize the improvement to the quality of the model fit achieved by the addition of the second component by the model evidence ratio evaluated through MultiNest of $e^{86} \sim 10^{37}$, which takes into account the additional parameter space available to the more general model. Therefore, we conclude that a single-component rotating disk model is not sufficient to describe the [C II] emission in CRLE, and we find, instead, a strong indication of a second component corresponding to the narrow line emission component also observed in the $\mathrm{CO}$ and [N II] lines. Higherresolution observations are required in order to determine if coherent rotation may be important to the gas dynamics in this system or whether the dynamics are dispersion-dominated. The latter would provide stronger evidence in favor of a merging pair of galaxies, perhaps identified by the two separate dynamical components. However, strong shocks and winds may also be responsible for skewing the velocity profile and could therefore contribute to the observed dynamics.

\section{ORCID iDs}

Riccardo Pavesi (1) https://orcid.org/0000-0002-2263-646X Dominik A. Riechers (ㄷ) https://orcid.org/0000-00019585-1462

Chelsea E. Sharon (1) https://orcid.org/0000-0002-6250-5608 Vernesa Smolčić (10) https://orcid.org/0000-0002-3893-8614

Andreas L. Faisst (1) https://orcid.org/0000-0002-9382-9832

Eva Schinnerer (10 https://orcid.org/0000-0002-3933-7677

Christopher L. Carilli (ib https://orcid.org/0000-0001-

6647-3861

Nick Scoville iㅣ https://orcid.org/0000-0002-0438-3323

\section{References}

Aravena, M., Bertoldi, F., Carilli, C., et al. 2010, ApJL, 708, L36 Barišić, I., Faisst, A. L., Capak, P. L., et al. 2017, ApJ, 845, 41 Bertin, E., \& Arnouts, S. 1996, A\&AS, 117, 393
Blain, A. W., Smail, I., Ivison, R. J., Kneib, J.-P., \& Frayer, D. T. 2002, PhR, 369,111

Bolatto, A. D., Wolfire, M., \& Leroy, A. K. 2013, ARA\&A, 51, 207

Bruzual, G., \& Charlot, S. 2003, MNRAS, 344, 1000

Buchner, J., Georgakakis, A., Nandra, K., et al. 2014, A\&A, 564, A125

Calzetti, D. 2001, PASP, 113, 1449

Capak, P. L., Carilli, C., Jones, G., et al. 2015, Natur, 522, 455

Capak, P. L., Riechers, D., Scoville, N. Z., et al. 2011, Natur, 470, 233

Carilli, C. L., Daddi, E., Riechers, D., et al. 2010, ApJ, 714, 1407

Carilli, C. L., \& Walter, F. 2013, ARA\&A, 51, 105

Casey, C. M., Narayanan, D., \& Cooray, A. 2014, PhR, 541, 45

Chen, C.-C., Hodge, J. A., Smail, I., et al. 2017, ApJ, 846, 108

Chiang, Y.-K., Overzier, R., \& Gebhardt, K. 2013, ApJ, 779, 127

Chiang, Y.-K., Overzier, R. A., Gebhardt, K., \& Henriques, B. 2017, ApJL, 844, L23

Cooray, A., Calanog, J., Wardlow, J. L., et al. 2014, ApJ, 790, 40

da Cunha, E., Groves, B., Walter, F., et al. 2013, ApJ, 766, 13

Danielson, A. L. R., Swinbank, A. M., Smail, I., et al. 2017, ApJ, 840, 78

Davis, T. A., Alatalo, K., Bureau, M., et al. 2013, MNRAS, 429, 534

Decarli, R., Walter, F., Venemans, B. P., et al. 2017, Natur, 545, 457

Delhaize, J., Smolčić, V., Delvecchio, I., et al. 2017, A\&A, 602, A4

Díaz-Santos, T., Armus, L., Charmandaris, V., et al. 2017, ApJ, 846, 32

Downes, D., \& Solomon, P. M. 1998, ApJ, 507, 615

Draine, B. T., \& Li, A. 2007, ApJ, 657, 810

Dunne, L., Eales, S. A., \& Edmunds, M. G. 2003, MNRAS, 341, 589

Engel, H., Tacconi, L. J., Davies, R. I., et al. 2010, ApJ, 724, 233

Faisst, A. L., Capak, P. L., Yan, L., et al. 2017, ApJ, 847, 21

Feroz, F., Hobson, M. P., \& Bridges, M. 2009, MNRAS, 398, 1601

Foreman-Mackey, D., Hogg, D. W., Lang, D., \& Goodman, J. 2013, PASP, 125,306

Geach, J. E., Dunlop, J. S., Halpern, M., et al. 2017, MNRAS, 465, 1789

Gómez-Guijarro, C., Toft, S., Karim, A., et al. 2018, ApJ, 856, 121

Groves, B. A., Schinnerer, E., Leroy, A., et al. 2015, ApJ, 799, 96

Harris, A. I., Baker, A. J., Frayer, D. T., et al. 2012, ApJ, 752, 152

Herrera-Camus, R., Bolatto, A., Smith, J. D., et al. 2016, ApJ, 826, 175

Herrera-Camus, R., Sturm, E., Graciá-Carpio, J., et al. 2018a, arXiv:1803. 04419

Herrera-Camus, R., Sturm, E., Graciá-Carpio, J., et al. 2018b, arXiv:1803. 04422

Higuchi, R., Ouchi, M., Ono, Y., et al. 2018, arXiv:1801.00531

Hodge, J. A., Carilli, C. L., Walter, F., et al. 2012, ApJ, 760, 11

Hodge, J. A., Riechers, D., Decarli, R., et al. 2015, ApJL, 798, L18

Ivison, R. J., Lewis, A. J. R., Weiss, A., et al. 2016, ApJ, 832, 78

Ivison, R. J., Papadopoulos, P. P., Smail, I., et al. 2011, MNRAS, 412, 1913

Ivison, R. J., Swinbank, A. M., Smail, I., et al. 2013, ApJ, 772, 137

Keeton, C. R. 2001, arXiv:astro-ph/0102340

Kennicutt, R. C., Jr. 1998, ApJ, 498, 541 
Kirkpatrick, A., Pope, A., Sajina, A., et al. 2015, ApJ, 814, 9 Koprowski, M. P., Dunlop, J. S., Michałowski, M. J., et al. 2017, MNRAS, 471, 4155

Laigle, C., McCracken, H. J., Ilbert, O., et al. 2016, ApJS, 224, 24

Leipski, C., Meisenheimer, K., Walter, F., et al. 2013, ApJ, 772, 103

Leipski, C., Meisenheimer, K., Walter, F., et al. 2014, ApJ, 785, 154

Lewis, A. J. R., Ivison, R. J., Best, P. N., et al. 2017, arXiv:1711.08803

Li, Y., Hernquist, L., Robertson, B., et al. 2007, ApJ, 665, 187

Lutz, D., Poglitsch, A., Altieri, B., et al. 2011, A\&A, 532, A90

Magdis, G. E., Daddi, E., Elbaz, D., et al. 2011, ApJL, 740, L15

Marrone, D. P., Spilker, J. S., Hayward, C. C., et al. 2018, Natur, 553, 51

Molnár, D. C., Sargent, M. T., Delhaize, J., et al. 2018, MNRAS, 475, 827

Murayama, T., Taniguchi, Y., Scoville, N. Z., et al. 2007, ApJS, 172, 523

Murphy, E. J., Condon, J. J., Schinnerer, E., et al. 2011, ApJ, 737, 67

Narayanan, D., Turk, M., Feldmann, R., et al. 2015, Natur, 525, 496

Noll, S., Burgarella, D., Giovannoli, E., et al. 2009, A\&A, 507, 1793

Oberst, T. E., Parshley, S. C., Stacey, G. J., et al. 2006, ApJL, 652, L125

Oteo, I., Ivison, R. J., Dunne, L., et al. 2016, ApJ, 827, 34

Oteo, I., Ivison, R. J., Dunne, L., et al. 2018, ApJ, 856, 72

Ouchi, M., Harikane, Y., Shibuya, T., et al. 2018, PASJ, 70, S13

Overzier, R. A., Guo, Q., Kauffmann, G., et al. 2009, MNRAS, 394, 577

Pavesi, R., Riechers, D. A., Capak, P. L., et al. 2016, ApJ, 832, 151

Peng, C. Y., Ho, L. C., Impey, C. D., \& Rix, H.-W. 2002, AJ, 124, 266

Riechers, D. A., Bradford, C. M., Clements, D. L., et al. 2013, Natur, 496, 329

Riechers, D. A., Capak, P. L., Carilli, C. L., et al. 2010, ApJL, 720, L131

Riechers, D. A., Carilli, C. L., Capak, P. L., et al. 2014a, ApJ, 796, 84
Riechers, D. A., Carilli, L. C., Walter, F., et al. 2011, ApJL, 733, L11

Riechers, D. A., Leung, T. K. D., Ivison, R. J., et al. 2017, ApJ, 850, 1 Riechers, D. A., Pope, A., Daddi, E., et al. 2014b, ApJ, 786, 31

Santini, P., Maiolino, R., Magnelli, B., et al. 2010, A\&A, 518, L154

Schinnerer, E., Sargent, M. T., Bondi, M., et al. 2010, ApJS, 188, 384

Scoville, N., Aussel, H., Brusa, M., et al. 2007, ApJS, 172, 1

Scoville, N., Lee, N., Vanden Bout, P., et al. 2017a, ApJ, 837, 150

Scoville, N., Murchikova, L., Walter, F., et al. 2017b, ApJ, 836, 66

Scoville, N., Sheth, K., Aussel, H., et al. 2016, ApJ, 820, 83

Scoville, N., Sheth, K., Walter, F., et al. 2015, ApJ, 800, 70

Serra, P., Amblard, A., Temi, P., et al. 2011, ApJ, 740, 22

Sharon, C. E., Riechers, D. A., Hodge, J., et al. 2016, ApJ, 827, 18

Shibuya, T., Ouchi, M., Konno, A., et al. 2018, PASJ, 70, S14

Silva, L., Granato, G. L., Bressan, A., \& Danese, L. 1998, ApJ, 509, 103

Smolčić, V., Miettinen, O., Tomičić, N., et al. 2017a, A\&A, 597, A4

Smolčić, V., Novak, M., Bondi, M., et al. 2017b, A\&A, 602, A1

Springel, V., White, S. D. M., Jenkins, A., et al. 2005, Natur, 435, 629

Stacey, G. J., Geis, N., Genzel, R., et al. 1991, ApJ, 373, 423

Strandet, M. L., Weiss, A., De Breuck, C., et al. 2017, ApJL, 842, L15

Strandet, M. L., Weiss, A., Vieira, J. D., et al. 2016, ApJ, 822, 80

Swinbank, A. M., Simpson, J. M., Smail, I., et al. 2014, MNRAS, 438, 1267

Tabatabaei, F. S., Schinnerer, E., Krause, M., et al. 2017, ApJ, 836, 185

Vallini, L., Pallottini, A., Ferrara, A., et al. 2018, MNRAS, 473, 271

Walter, F., Decarli, R., Carilli, C., et al. 2012, Natur, 486, 233

Weiß, A., De Breuck, C., Marrone, D. P., et al. 2013, ApJ, 767, 88 